\title{
Investigation of the structures in the unstable rotating-cone boundary layer
}

\author{
K. Kato, ${ }^{1, *}$ T. Kawata, ${ }^{2, \dagger}$ P. H. Alfredsson,,${ }^{1,}$ and R. J. Lingwood ${ }^{1,3, \S}$ \\ ${ }^{1}$ Linné FLOW Centre, KTH Mechanics, SE-100 44 Stockholm, Sweden \\ ${ }^{2}$ Department of Mechanical Engineering, Tokyo University of Science, 2641 Yamazaki, \\ Noda, Chiba 278-8510, Japan \\ ${ }^{3}$ Department of Mechanical and Aerospace Engineering, Brunel University London, \\ UB8 3PH, United Kingdom
}

(Received 14 December 2018; published 30 May 2019)

\begin{abstract}
This work reports on the unstable region and the transition process of the boundary-layer flow induced by a rotating cone with a half apex angle of 60 degrees using the probability density function (PDF) contour map of the azimuthal velocity fluctuation, which was first used by Imayama et al. [Phys. Fluids 24, 031701 (2012)] for the similar boundary-layer flow induced by a rotating disk. The PDF shows that the transition behavior of the rotatingcone flow is similar to that on the rotating disk. The effects of roughness elements on the cone surface have been examined. For the cone with roughnesses, we reconstructed the most probable vortex structure within the boundary layer from the hot-wire anemometry time signals. The results show that the PDF clearly describes the overturning process of the high-momentum upwelling of the spiral vortices, which due to vortex meandering cannot be detected in the phase-averaged velocity field reconstructed from the point measurements. At a late stage of the overturning process, our hot-wire measurements captured high-frequency oscillations, which may be related to secondary instability.
\end{abstract}

DOI: 10.1103/PhysRevFluids.4.053903

\section{INTRODUCTION}

Flows induced by rotating cones and disks have been investigated as generic models of threedimensional boundary layers, e.g., flow over a swept wing [1], flow on turbomachinery blades [2], and flows related to some chemical applications such as desalination [3] or condensation [4]. The rotating-cone flow is the general case (Fig. 1) where the rotating-disk flow is a special case with a cone angle of $\psi=90^{\circ}$. When the cone angle is large $\left(50^{\circ} \lesssim \psi \leqslant 90^{\circ}\right)$, the laminar boundary layer developing from the apex (or center in the case of a disk) is susceptible to an inviscid cross-flow instability, whereas for small cone angles $\left(\psi \lesssim 30^{\circ}\right)$, the flow is susceptible to centrifugal instability ( $\psi=0^{\circ}$ corresponding to the flow induced by a rotating cylinder) [5]. Thus, the rotating-cone flow is a fundamental and interesting flow manifesting different instabilities depending on the cone angle.

Here, we consider a cone rotating at a rotational rate $\Omega^{*}$ in still fluid $(*$ denotes a dimensional quantity). An orthogonal coordinate system $(x, \theta, z)$ is defined as shown in Fig. 1, where $x$ and $z$ are, respectively, the coordinates along the generating line of the cone and the wall-normal direction with the origin located at the apex, and are nondimensionalized by a viscous length, $\delta_{v}^{*}=\sqrt{v^{*} /\left(\Omega^{*} \sin \psi\right)}$, where $v^{*}$ is the kinematic viscosity of the surrounding fluid. $\theta$ is the

\footnotetext{
*kentaro.kato@mech.kth.se

†kawata@rs.tus.ac.jp

†phal@mech.kth.se

\$rebecca.lingwood@brunel.ac.uk
} 


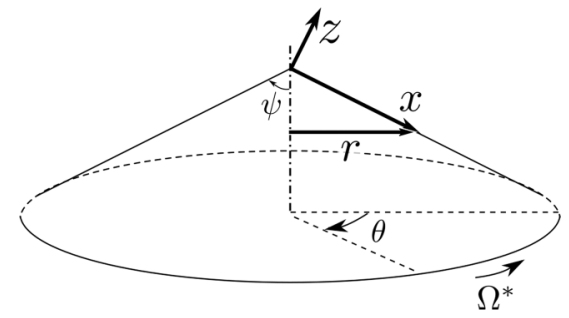

FIG. 1. The coordinate system $(x, \theta, z)$ for the rotating cone. We also define a radius $r=x \sin \psi$. The velocity in the azimuthal direction is denoted as $V+v$ where $V(x, z)$ is the averaged velocity in $\theta$ (and time) and $v(t ; x, z, \theta)$ is the deviation from $V$ at a given position $(x, z, \theta)$ as function of time. The time and $\theta$ average of $v$ is zero, but the time average of $v$ is not in general zero at a fixed position in space.

azimuthal coordinate in a frame rotating with the cone surface. When the cone is rotating a boundary layer forms where the fluid is forced to move with the cone in the azimuthal direction due to the no-slip condition at the surface, but it is also transported in the $x$ direction, hence the cone acts as a centrifugal pump. To compensate for the flow in the $x$ direction, fluid is drawn toward the cone surface.

The flow on a rotating disk is known as the von Kármán disk flow. In his seminal paper [6], von Kármán showed that there exists a similarity solution for the laminar boundary layer on the rotating disk. A similarity solution can also be obtained for the rotating-cone case as shown by Ref. [7], which (using the viscous length $\delta_{v}^{*}$ as a scaling parameter) gives the same similarity equations as those derived by von Kármán for the rotating disk. The length along the cone surface is nondimensionalized as $x=x^{*} / \delta_{v}^{*}$ and the square of this value can be seen as a Reynolds number,

$$
\operatorname{Re}=\frac{\Omega^{*} x^{* 2} \sin \psi}{v^{*}}
$$

On the wider cone $\left(50^{\circ} \lesssim \psi \leqslant 90^{\circ}\right)$, the flow is susceptible to inviscid cross-flow instability [8,9] at sufficiently high Reynolds numbers. This instability gives rise to slightly inclined disturbances with respect to the azimuthal direction and grow with increasing $x$ to become co-rotating spiral vortices. The disturbances most often and easily observed are stationary with respect to the rotating surface (i.e. fixed in $\theta$ for a specified $x$ ) because, unless a particular time-dependent disturbance is artificially introduced, the disturbances are primarily triggered continuously by unavoidable surface roughness. For increasing $x$ (or Re) the disturbance amplitude saturates and the stationary vortices "collapse" leading to transition from a laminar to a turbulent boundary layer. These vortices are quite similar to those observed in swept-wing boundary layer $[10,11]$. In the recent work by Groot et al. [11] a comprehensive collection of references to swept-wing boundary-layer stability and transition can be found.

The stability of the boundary layer on the rotating disk was analyzed by Lingwood $[12,13]$ who found an absolute instability, which was proposed to be responsible for the particular transition Reynolds number found in different experimental studies at that time on smooth disks, although on rough disks transition will occur at a lower Reynolds numbers [14]. This has led to further studies both through experiments (e.g. [15]) and direct numerical simulations [16] of the disk flow (an extensive list of the relevant literature for the rotating disk can be found in $[15,16]$ and also in a recent review [17]).

The recent simulations by Appelquist et al. [16] were able to shed some further light on the transition scenarios on the disk. In the simulations the disturbances were triggered by a stationary forcing at the wall (imitating discrete surface roughness) and two different transition scenarios were found, depending on the height of the surface roughnesses or rather the amplitude of the stationary forcing. With low-amplitude forcing, the onset of secondary instability was observed before the amplitude of the cross-flow vortices reached the saturation level but the flow first became turbulent 
after the primary disturbance amplitude reached the saturation level. In the case of high-amplitude forcing, the primary disturbance amplitude reached the saturated level before the onset of the secondary absolute instability, and the flow underwent transition at the critical Reynolds number for the secondary global instability. In the latter case, compared with the former, the secondary instability was observed over a wider range of Reynolds numbers, especially where the spatial gradients of the mean velocity are large.

These cases with low/high-amplitude forcing correspond to experiments without/with artificial roughness elements $[15,18]$, typically with a height of some micrometers, i.e. about two orders of magnitude smaller than $\delta_{v}^{*}$. However, the secondary instability has not been directly observed in experiments although some flow visualization photographs indicate the appearance of small wavelength disturbances on top of the stationary vortices (e.g., Fig. 8 in Ref. [19]) and some kinks in measured time signals are reported (e.g., Fig. 11 in Ref. [18]).

For the transition on the clean disk, an interesting method describing the transition stages was proposed by Imayama et al. [20]. The method graphically shows different flow characteristics of the transition using the normalized probability density function (PDF) contour map of the azimuthal velocity fluctuation. The PDF method clearly illuminates changes in the wall-normal structure of the flow. They inferred that a topological change in the PDF around $2.0 \leqslant z \leqslant 2.8$ (Fig. 7 in Ref. [20]) may represent a secondary instability. However, as yet, it has not been shown conclusively whether this change corresponds to a structural change in the spiral vortices or to a secondary instability.

This paper is the first experimental work to report on the detailed changes the cross-flow vortices on the cone undergo as they develop. Through measurements of the azimuthal velocity on a cone with $\psi=60^{\circ}$, for which the inviscid cross-flow instability is expected to be the primary instability (similar to the disk flow), this work aims to address the following issues: (i) effects of the micrometer-sized artificial (regularly spaced in the azimuthal direction) roughness elements on the PDF; (ii) interpretation of the PDF, especially the topological change of the PDF with respect to the vortex structure; (iii) reconstruction of the vortex structure during its development in $x$; and (iv) exploration of the experimental data to find signs of the secondary instability. In addition, the paper provides a comparison of the cone case with the disk case and comparisons between the PDF and other common measures describing the transition, e.g., root-mean square (rms) and Fourier power spectra of the velocity fluctuations.

The paper is organized as follows. Section II briefly describes the experimental setup and methodology and Sec. III describes and discusses the experimental results. It is divided into four subsections: Sec. III A shows the mean flow and rms profiles on the cone, whereas Sec. III B shows the PDF plots for two different cone cases and makes a comparison with two previously measured disk cases. In Sec. III C, a method that allows the vortex structures to be reconstructed from single point data are presented and the resulting vortex structures are discussed in detail. Section III D shows results where the secondary instability is detected in the some velocity signals and how it is related to vortex meandering. Finally, Sec. IV gives the conclusion of the work. Appendix A describes in some detail a new calibration procedure developed for the hot wires, Appendix B provides a comparison of two different rotational speeds to verify that Reynolds number similarity is valid and Appendix $\mathrm{C}$ gives some examples of merging and splitting of vortices on the clean cone.

\section{EXPERIMENTAL SET-UP}

The rotating-cone facility consists of a precision-made solid aluminium alloy cone mounted on an air bearing and driven by a d.c.-motor. The cone has a base diameter of $474 \mathrm{~mm}$. The cone surface was smoothly finished (resulting in a surface roughness of approx. $1 \mu \mathrm{m}$ ). The cone was accurately aligned and rotated on the vertical axis (the rotational imbalance was approx. $10 \mu \mathrm{m}$ at the edge). At the edge of the cone, a fixed, horizontal wooden annular plate was positioned flush with the cone surface. The cone was spun at a rotational speed of $900 \mathrm{rpm}$. This rotational speed 


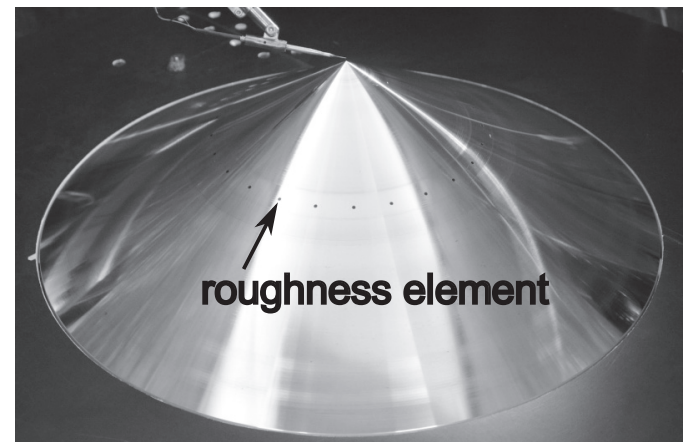

FIG. 2. The 60-degree cone with 24 artificial roughness elements that are uniformly mounted on the cone surface at $x^{*}=115.7 \pm 0.5 \mathrm{~mm}$. At the top of the figure, the hot-wire probe can be seen. Cone rotation is anti-clockwise.

was chosen so that transition occurs far enough away from the edge of the cone $(x=629)$ while the vortex structures can still be sufficiently resolved spatially within the boundary layer.

A single hot-wire probe with its sensing element parallel to the $x$-direction was used to measure the azimuthal velocity component. The hot wire has a sensing length of approx. $0.5 \mathrm{~mm}$ and a diameter of $2.5 \mu \mathrm{m}$. The probe was moved by means of a two-axes traverse system in the $x$ - and $z$-directions. The velocity was measured at fixed points in the laboratory frame. The signals from a Constant Temperature Anemometer (CTA) and the tachometer of the driving system were recorded simultaneously during approx. $60 \mathrm{~s}$ at a sampling rate of 720 data points per cone revolution. In the following the measured velocity was normalized by the local wall velocity $V_{\text {wall }}^{*}=\Omega^{*} x^{*} \sin \psi$. From the simultaneous recordings, it is possible to reconstruct stationary flow structures. For this kind of analysis the velocity signal was post-processed using a high-pass filter $\left(\omega^{*} / \Omega^{*}>15\right)$ for $x<510$ on the clean cone and for $x<430$ on the cone with roughnesses, where $\omega^{*}$ is the disturbance angular frequency (in the laboratory frame). Note that if the vortices are fixed with respect to the cone surface, then $\omega^{*} / \Omega^{*}=\omega$ gives the azimuthal wavenumber.

Further details of the driving system and data acquisition can be found in Refs. [20,21]. Compared with Refs. [20,21], two changes were made when the disk was replaced by the cone: (a) the traversing system was separated from the driving system and was placed directly on the laboratory floor; and (b) some electrical changes to the electrical configuration were made (introducing ferrite cores and shielding the cables). These changes improved the noise-signal ratio and helped to better resolve the early development of the flow instability when the amplitudes were low.

For accurate calibration and positioning of the hot wire in the boundary layer, a new calibration methodology was developed. In the work on the rotating disk by Imayama et al. [20] the calibration of the hot wire was completed using the laminar boundary layer profile by first optically determining the position of the probe accurately with respect to the disk surface. However, for the cone case the optical determination of distance from the wall was less accurate due to the curved surface; instead the calibration curve and the distance from the cone surface to the hot wire was simultaneously obtained based on a least-square method from measurements in the laminar boundary layer. The details can be found in Appendix. A.

To introduce reproducible stationary disturbance, artificial roughness elements were mounted on the cone surface. As shown in Fig. 2, 24 artificial roughness elements were mounted uniformly in the azimuthal direction (at angular intervals of $15 \pm 0.3^{\circ}$ ) at $x^{*}=115.7 \pm 0.5 \mathrm{~mm}$ corresponding to $x=267$. The number of 24 was chosen based on the measurements taken for the clean cone (the results are discussed with Fig. 6) so that vortex structures similar to those seen without roughness elements can be studied. The roughness elements are located slightly upstream of the neutral 
stability point so that the harmonics introduced by the roughness decay and the first-amplified disturbance consists entirely of the fundamental component. Dry transfer lettering provided by Letraset (Letraset Ref. No. 13045) was used to create the roughness elements, which was also the case for Ref. [15]. Each element has a circular shape with a diameter and height of approximately $2 \mathrm{~mm}$ and $4 \mu \mathrm{m}$, which correspond to about $5 \delta_{v}^{*}$ and $0.01 \delta_{v}^{*}$, respectively.

\section{RESULTS AND DISCUSSIONS}

\section{A. Mean and rms profiles}

The profiles of the time-averaged azimuthal velocity component $V$ at different $x$ locations are shown in Fig. 3. For the clean-cone case (a) and cone with roughnesses (b), the measured mean profiles (circles) are seen to agree with the similarity solution (solid curves) up to $x=498$ and $x=461$, respectively. Upstream of these positions, the $90 \%$ boundary-layer thickness $\delta_{90}^{*}$, where $V$ becomes $10 \%$ of $V_{\text {wall }}^{*}$, also agrees with that based on the similarity solution, i.e., $z=2.81$. Below $x=498$ and $x=461$ without and with roughnesses, the difference between the measured mean velocity and the similarity solution does not exceed $5 \%$ of the local wall velocity. The artificial roughnesses do not seem to affect the time-averaged laminar profiles although the roughnesses promote transition. Beyond the laminar regime, the boundary-layer thickness increases significantly and the profile approaches the typical turbulent profile.

A corresponding behavior is also seen in the rms of the azimuthal velocity $v_{\text {rms }}$ in Fig. 4. In the laminar regime, one peak of $v_{\text {rms }}$ is observed, which is caused by the spiral cross-flow vortices. During the transition process the boundary layer thickens, and the peak of $v_{\text {rms }}$ becomes broader and extends further into the outer layer. To further investigate the process of transition to turbulence, we use the PDF approach in the same manner as Ref. [20] in Sec. III B, where we compare the transition process on the cone with that on the disk using the PDF map of $v$ around the local maximum of $v_{\text {rms }}$ (as shown by the dotted line in Fig. 4).

\section{B. PDF and the vortex structure}

Figure 5 shows four different cases (two disk cases based on data from Refs. [15,20] and two cases from the cone of the present study) where the PDF map is used to analyze the transition scenario. We use Fig. 5(a) to describe the general features of the PDF map, as introduced by Ref. [20] to analyze the transition process. The data are taken from case I01 and Fig. 6 in Ref. [20], and Fig. 5(a) here shows the PDF of the azimuthal velocity fluctuation $v$ at $z=1.3$ for a case without any artificial roughnesses. At each $x$ location, the PDF is normalized by the local maximum. In the early laminar stage, the PDF map is narrow. Beyond $x=475$ (this lower limit depends on the noise level and bin size of the PDF), the transition process can be classified into four stages according to the PDF as shown in Fig. 5(a):

(i) Linear and weakly nonlinear stage $(475<x<540)$ : The PDF contours spread nearly exponentially.

(ii) Strongly nonlinear stage $(540<x<565)$ : At the beginning of this stage, the slope of the outer contour of the PDF changes suddenly and PDF begins to be skewed.

(iii) Saturation and collapse stage $(565<x<600)$ : The skewness is positive and decreases at the end of this stage. Around the middle of this stage, the width of the PDF reaches its maximum.

(iv) Turbulent stage $(x>600)$ : The PDF gradually approaches fully developed turbulent one.

This classification can be connected to characteristic stages of the transition process on the disk, summarized in Fig. 14 of Ref. [18]. In stage (i), the mean flow is still close to the similarity solution although the first harmonic in the spectrum has already appeared by $x=510$, indicating the onset of the nonlinearity. In stage (ii), the mean flow begins to change and nonlinear saturation of stationary disturbances occurs at $x=550$. On the border between stages (ii) and (iii), $x=565$, the onset of secondary instability and rapid growth of high-frequency components are reported. At $x=570$, the 

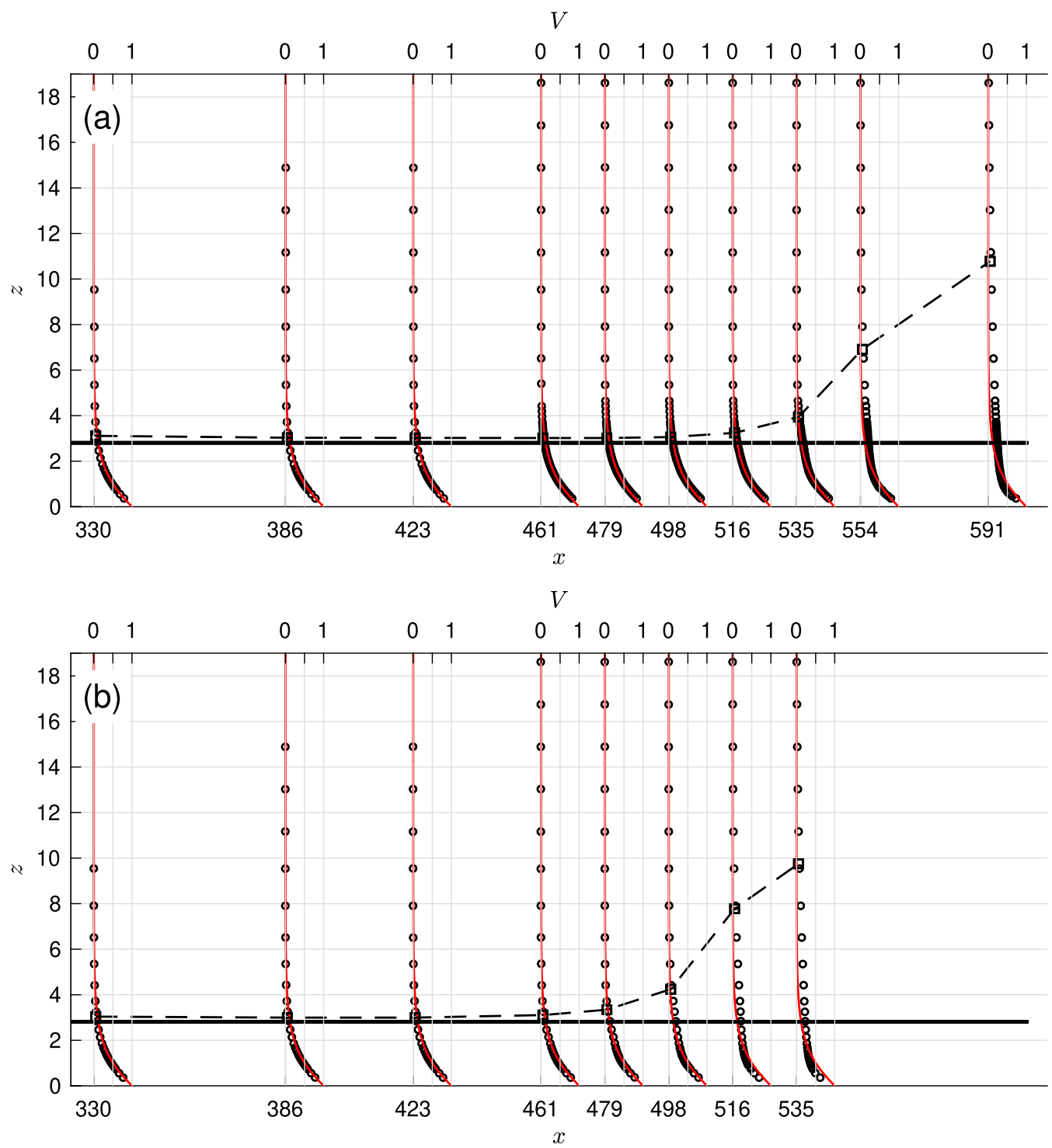

FIG. 3. Profiles of azimuthal mean velocity $V$ (circles) in the laboratory frame (a) without and (b) with roughnesses at $x=267$. The solid curves show the similarity solution. The thick line at $z=2.81$ indicates the $90 \%$ boundary-layer thickness $\delta_{90}$ for the similarity solution, where $V$ becomes 0.10 . The squares with the dashed lines show the measured $90 \%$ boundary-layer thickness.

onset of turbulent breakdown occurs. Through the saturation of total disturbance at $x=585$, the flow approaches fully developed turbulence $(x=650)$ in stage (iv).

The other cases shown in Fig. 5 compare the PDF with the classification mentioned above. Figure 5(b) shows the case for the clean cone $\left(\psi=60^{\circ}\right)$ rotating at $900 \mathrm{rpm}$. It show a good agreement with the clean disk in (a) except for stage (ii) (the reason for the sensitivity of the sign of the skewness will be explained by Fig. 7). Figures 5(c) and 5(d) show cases for the cone and disk with artificial roughness elements mounted uniformly in the azimuthal direction. The data for Fig. 5(d) were taken from Ref. [15], corresponding to the case IP01R32, and has not been shown as a PDF map before. In Figs. 5(a) and 5(b), the transition locations measured by Kobayashi and Izumi 

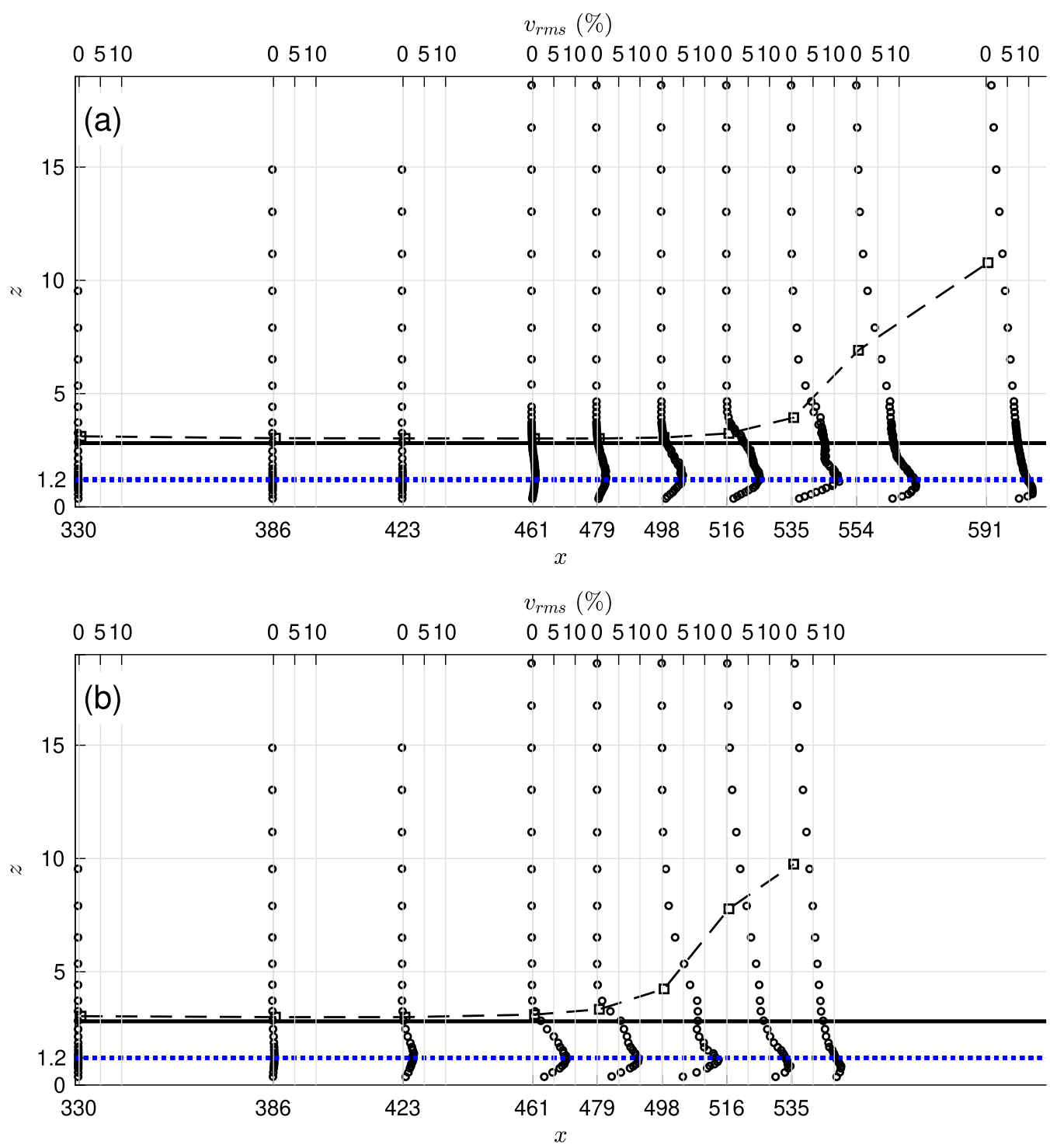

FIG. 4. Profiles of rms of azimuthal velocity $v_{\text {rms }}$ (circles) (a) without and (b) with roughnesses at $x=267$. The thick line at $z=2.81$ indicates the $90 \%$ boundary-layer thickness for the similarity solution, where $V$ becomes 0.10 . The squares with the dashed lines show the measured $90 \%$ boundary-layer thickness. The dotted lines indicate the height $z=1.2$ where measurements for Figs. 5(b) and 5(c) were conducted.

[22] are also shown by dashed lines as another reference $\left(x=\sqrt{\operatorname{Re}}=5.7 \times 10^{2}\right.$ and $\left.5.2 \times 10^{2}\right)$. Both their work and our measurements show that transition occurs earlier on the cone as compared to the disk. As is well known for the rotating-disk case, stability and transition are functions of the Reynolds number and not the rotational speed per se. The same holds for the cone as can be seen in Appendix B.

The effect of the artificial roughness elements can be seen clearly in stage (i) when comparing the different cases in Fig. 5. The roughnesses mounted uniformly in the azimuthal direction give rise to uniform vortices resulting in a sinusoidal time signal, shown as symmetric double maxima for 

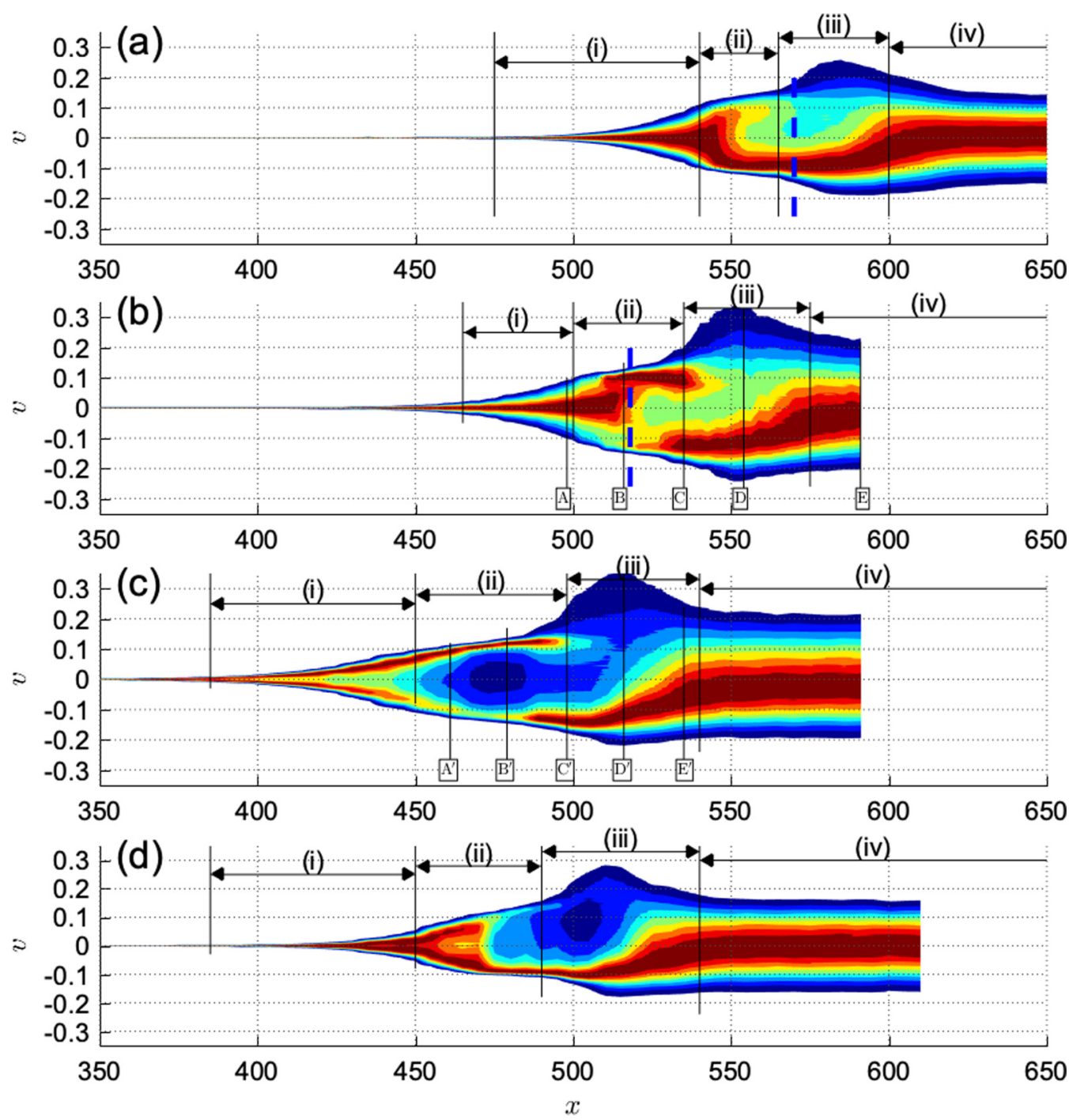

FIG. 5. The PDF of the azimuthal velocity fluctuation $v$ : (a) clean disk at $z=1.3,1400 \mathrm{rpm}$ from Ref. [20], (b) $60^{\circ}$ clean cone at $z=1.2,900 \mathrm{rpm}$, (c) $60^{\circ}$ cone with roughness elements at $z=1.2,900 \mathrm{rpm}$ and (d) disk with 32 roughness elements at $z=1.3,1013 \mathrm{rpm}$ from Ref. [15]. In (c) and (d), the roughness elements are located at $x=267$ and 287, respectively. Filled contours indicate 10\%, 20\%, 30\%, 40\%, 50\%, 60\%, 70\%, $80 \%$, and $90 \%$ of the local maxima of PDF. Dashed lines (a) and (b) indicate transition locations reported in Ref. [22]. The alphabetical symbols indicate the sections shown in Fig. 7.

positive and negative $v$ in Figs. 5(c) and 5(d). In contrast, the nonuniform roughnesses on the clean cone and disk result in an amplitude-modulated time signal, giving a single maxima around $v=0$ in (b) and (a) (see Fig. 9(a) in Ref. [18]). In the later stages (iii) and (iv), all cases show similar PDFs and no significant effect of the roughness elements was observed in the PDF.

As another characterization, Fourier power spectra from time signals measured on the cone at $z=$ 1.2 are shown in Fig. 6. Here, we calculated the spectrum with a frequency resolution of $\Delta \omega^{*} / \Omega^{*}=$ 0.2 based on Welch's method with a segment length of 5 cone revolutions and $0 \%$ overlap. (The time signal was split into segments and the spectrum was calculated for each segment. Finally, 

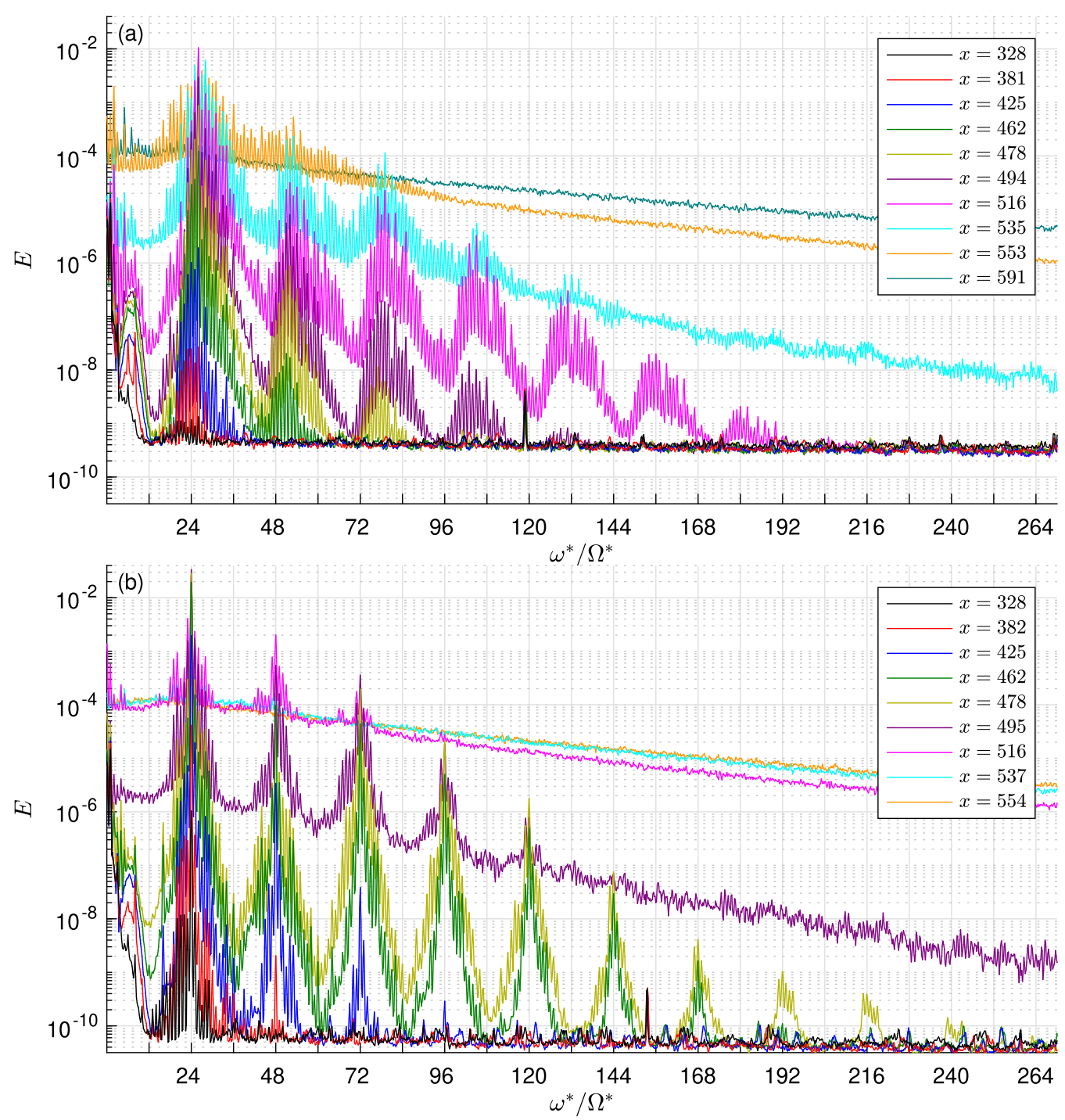

FIG. 6. Fourier power spectra $E$ of azimuthal velocity component $v$ at $z=1.2$ for different $x$ locations on (a) clean cone and (b) cone with roughness elements: $\omega^{*}$ indicates the disturbance angular frequency in the laboratory frame. Thus, for vortices fixed with respect to the cone surface, $\omega^{*} / \Omega^{*}$ indicates the azimuthal wave number.

these spectra were averaged.) The power spectral density $E$ of the fluctuation $v$ (without highpass filtering) is shown for different $x$ locations on the clean cone (a) and the cone with roughness elements (b). On the clean cone, a broad peak for $12 \lesssim \omega^{*} / \Omega^{*} \lesssim 36$ is observed at $x=328$ for which the amplitude increases with $x$. This broad peak contains spikes at integer values of $\omega^{*} / \Omega^{*}$, indicating the stationary disturbances with respect to the cone surface, namely, the stationary spiral vortices. Similar spikes were also observed on the clean disk in Refs. [18,23]. Initially, the dominant wave number in the peak changes within a range of 20 to 26 in repeated measurements with the same setup (probably due to the slight change of the surface roughness on the clean cone). From this observation, we determined the number of roughness elements as 24 . At $x \approx 462$, the first harmonic $\left(40 \lesssim \omega^{*} / \Omega^{*} \lesssim 60\right)$ appears, indicating the onset of nonlinearity. As $x$ increases, further 

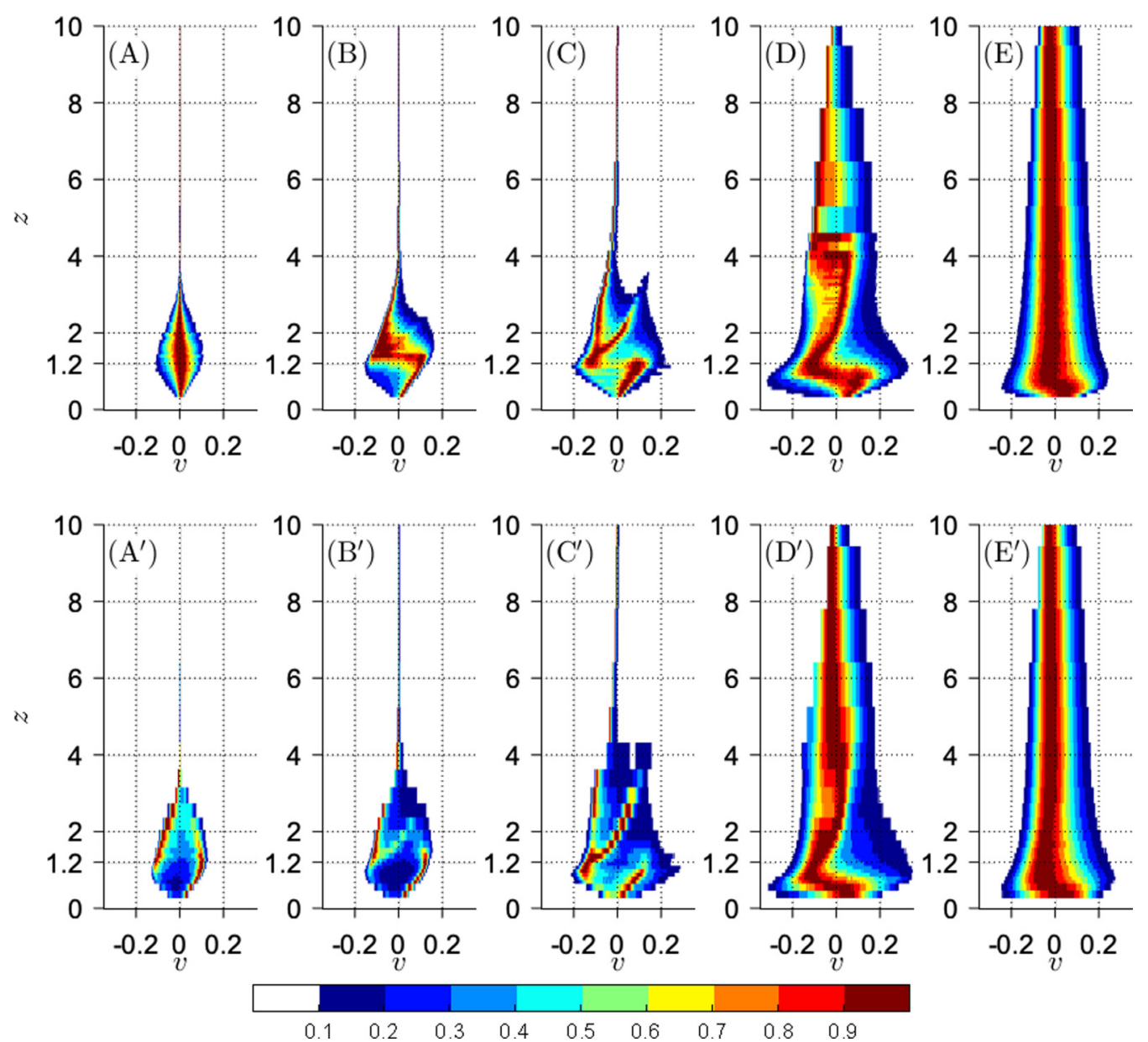

FIG. 7. The PDF of the azimuthal velocity fluctuation $v$ showing the $z$ structure at different $x$ locations: (A) $x=498$, (B) $x=516$, (C) $x=535$, (D) $x=554$, (E) $x=591$ on the clean cone [cf. Fig. 5(b)], and (A') $x=461,\left(\mathrm{~B}^{\prime}\right) x=479,\left(\mathrm{C}^{\prime}\right) x=498,\left(\mathrm{D}^{\prime}\right) x=516,\left(\mathrm{E}^{\prime}\right) x=535$ on the cone with roughness elements [cf. Fig. 5(c)].

higher harmonics appear. At $x=535$, the higher harmonics at $\omega^{*} / \Omega^{*}>140$ begin to disappear and the spectrum becomes smooth. On the cone with roughness, the sharper peak initially appears at $\omega^{*} / \Omega^{*}=24$, which demonstrates the sensitivity of the initially most-amplified wave number to the roughness on the surface. At $x=328$, no harmonics are observed. The first harmonic at $\omega^{*} / \Omega^{*}=48$ is seen at $x=382$. As $x$ increases, further higher harmonics appear at multiples of $\omega^{*} / \Omega^{*}=24$. Thus, the basic trends between the clean and artificially excited cones are similar although the transition location shifts and the dominant wave number changes slightly.

To capture the flow structure normal to the wall, the PDF of $v$ is calculated as a function of $z$ and the results are shown at some different $x$ locations in Fig. 7. The figure shows PDFs at the sections marked by (A-E) for the clean cone in Fig. 5(b) and $\left(\mathrm{A}^{\prime}-\mathrm{E}^{\prime}\right)$ for the cone with roughness elements in Fig. 5(c).

Each column of Fig. 7 shows a similar PDF structure although the $x$ locations are shifted to lower values for the cone with roughness elements. The $x$ positions for the roughness case were chosen to show similar PDF structures in both cases. The spatial resolution (in $z$ ) of the plots for the case with roughness is lower than for the clean cone case, which is due to fewer measurement points. In 
Fig. 7(A), the PDF is symmetric around the mean value within the boundary layer. In (B) and (A'), the lower $(z \lesssim 1.2)$ and upper parts $(z \gtrsim 1.2)$ of the boundary layer show negative and positive skew, respectively, indicating downwelling low-momentum fluid at low $z$ and upwelling high-momentum fluid at high $z$ due to the spiral vortices. It should be noted that the symmetric PDF is observed only in a narrow range around $z \approx 1.2$. If the measurement position is slightly shifted toward larger (smaller) $z$, the PDF would have a positive (negative) skewness. This sensitivity affects the sign of the skewness in stage (ii) of Fig. 5.

In $(C),\left(B^{\prime}\right)$, and $\left(C^{\prime}\right)$, double maxima appear for $z \gtrsim 1.2$. In $(\mathrm{D})$ and $\left(\mathrm{D}^{\prime}\right)$, where the breakdown has started, the branched maximum extends to the upper layer. In $(\mathrm{E})$ and $\left(\mathrm{E}^{\prime}\right)$, the PDF becomes smoother. A similar structure is also observed in the clean disk case (Fig. 7 in Ref. [20]).

To further understand the origin of the double maxima in Figs. $7(\mathrm{C}), 7\left(\mathrm{~B}^{\prime}\right)$, and $7\left(\mathrm{C}^{\prime}\right)$, the azimuthal velocity fluctuation $v$ is assessed at two different $x(x=479$ and 498) in Figs. 8 and 9 for the case with artificial roughness (corresponding to sections denoted by $\mathrm{B}^{\prime}$ and $\mathrm{C}^{\prime}$ in Figs. 5 and 7 ). The gray contour plots on the right-hand side of the figures show $v$ for the whole sample. Here, the ordinate gives the angle along the cone whereas the abscissa shows the cone revolution number. The measured velocity is plotted vertically (with a black/white color scale). and for the next revolution the plotting is starting over. The left-hand curves in the figures show both the phase-averaged signal for all revolutions (thick black line) and velocity signals for three consecutive revolutions (for the revolution numbers 300-302; these revolutions are picked more or less arbitrarily, similar results would be obtained for other choices as well; they are shown by the thin colored lines) at three different $z$. Note that the signals for the different $z$ are not simultaneous. In each figure, only data for a quarter of a cone $\left(0^{\circ} \leqslant \theta \leqslant 90^{\circ}\right)$ are shown.

The data for the three revolutions indicate that the branched maximum in $z \gtrsim 1.2$ at $x=498$ in Fig. 7(C') is caused by a plateau in the $v$-signal seen for the three revolutions in Figs. 9(b) and 9(c). As $z$ increases, the plateau is observed at higher values of $v$. Therefore, the branched maximum in Fig. 7( $\left.\mathrm{C}^{\prime}\right)$ shifts toward higher $v$. A similar plateau or small kink is also shown in Fig. 8(b) although it is not as clear. According to Fig. 7, this feature seems to be common both in the cases with and without roughnesses.

The gray contours on the right of Figs. 8 and 9 show that the spiral vortices actually move in the azimuthal direction during the cone revolutions although they are usually denoted as "stationary" vortices. Interestingly, all vortices shift in the same way simultaneously and the number of vortices remains the same as the number of roughnesses, which is consistent with the sharp peak in the spectra shown in Fig. 6(b). The red line on each contour plot of Figs. 8 and 9 shows the location of the vortices. The location was detected in the following way. First, the time signal was divided into 24 segments of 15 degrees each. Then, we took the phase-average of the 24 segments, and found the minimum and the corresponding angle, which is denoted as the vortex location. This vortex detection was completed for all revolutions. To make sure not to misinterpret a minimum that leaves the 15-degree segment and enters on the other side of the segment, the distance between minima for two consecutive revolutions is minimized. The shift can be of the order of $\Delta \theta=10^{\circ}$ even though the origin of each vortex is fixed by an artificial roughness. The typical timescale of this vortex meandering is of the order of 10 to 100 revolutions. The location of the vortices is used for the reconstruction of the most probable vortex structure in the next section.

In contrast, on the clean cone the number of vortices varies through merging and splitting of the vortices (see Appendix B for details). In the clean case, the origins of the vortices are not uniformly distributed in $x$ or in $\theta$. Furthermore, their amplitude (albeit small) can also vary. This results in a broad spectral peak as seen in Fig. 6(a). Because of the meandering of the spiral vortices, the plateau related to the branched maximum is smoothed out in the phase-averaged velocity.

\section{Reconstruction of the vortex structures}

To understand what the plateau in the velocity signal (described in the previous section) indicates, we reconstructed the most probable spiral vortex structure from the time signal of the hot-wire 
(a)
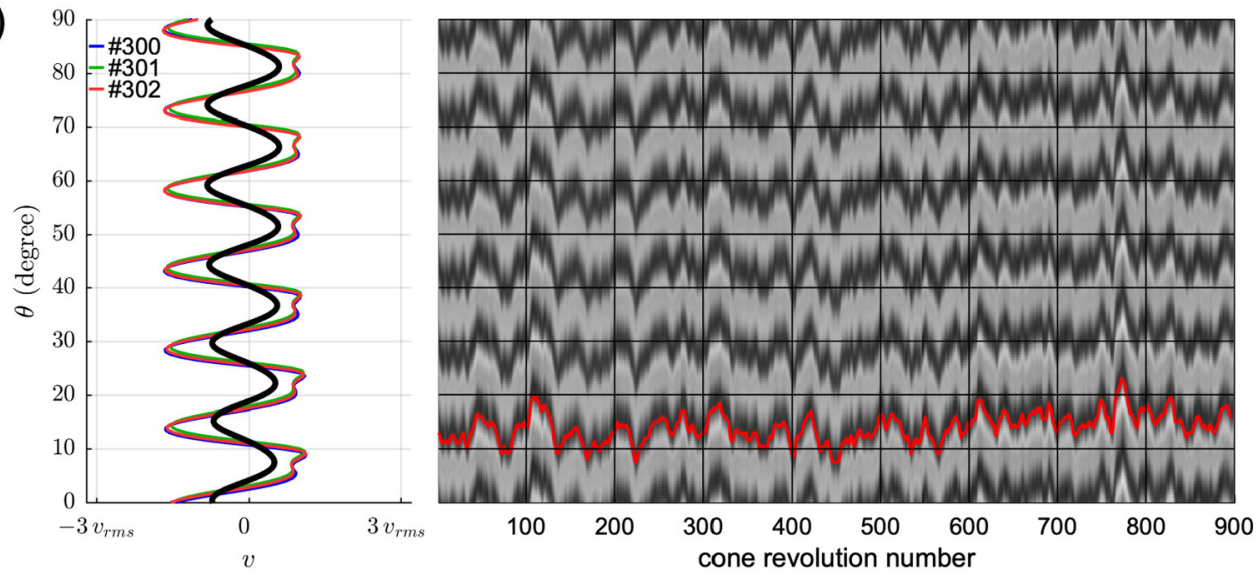

(b)
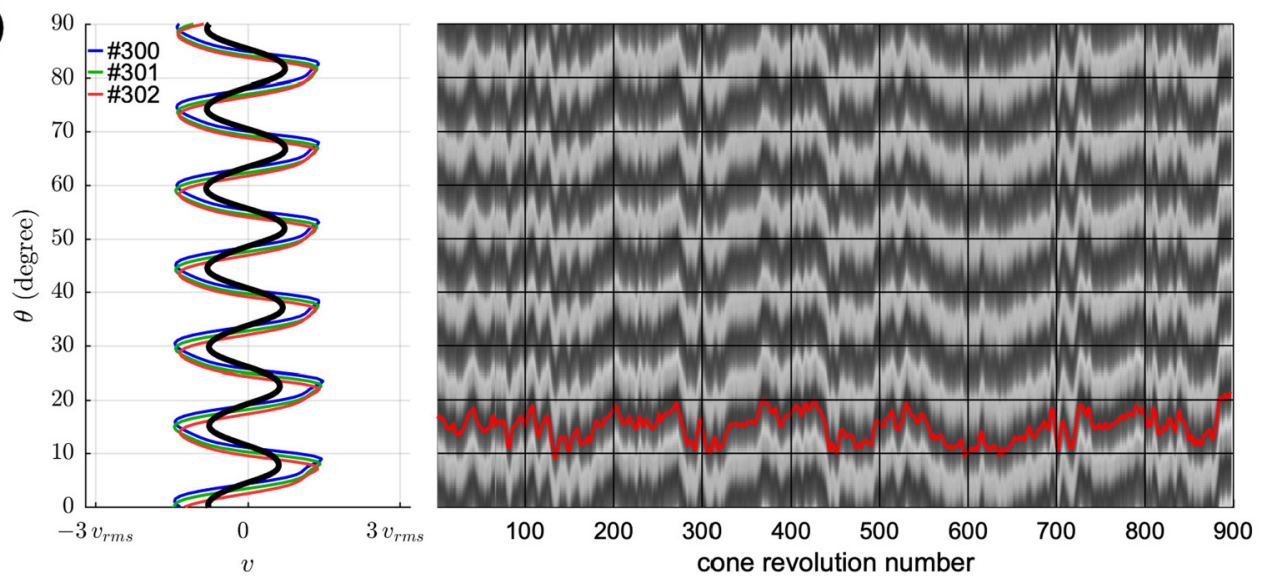

(c)
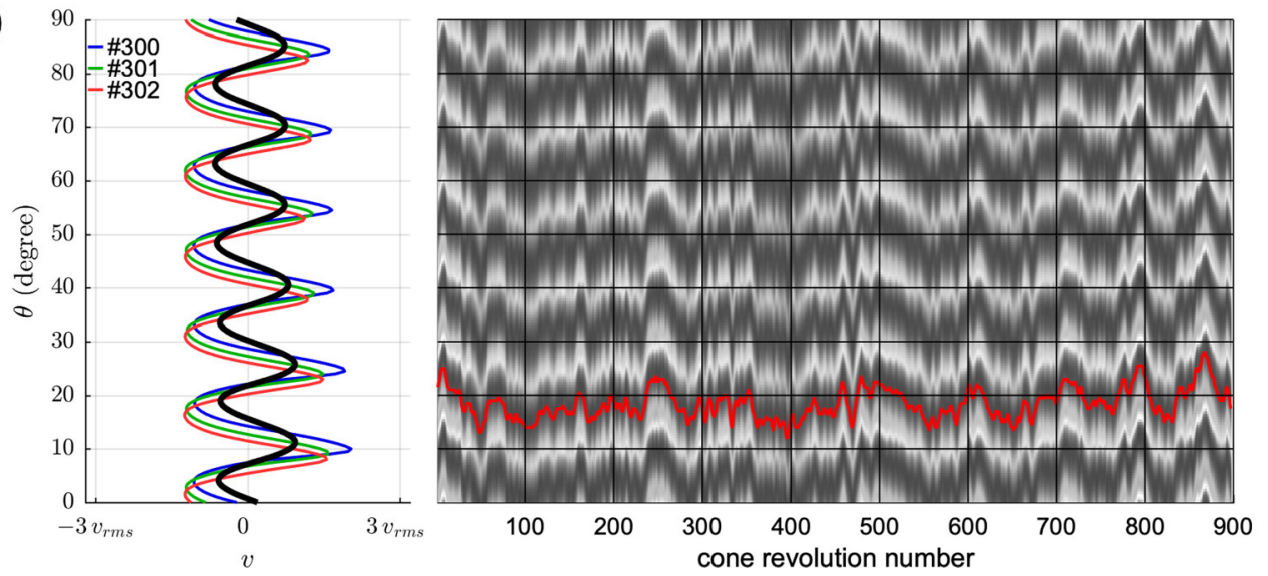

FIG. 8. Velocity fluctuation $v$ for single revolutions (thin colored lines to the left; blue, green, and red lines indicate $v$ for revolution numbers 300,301, and 302, respectively), phase-averaged velocity $\tilde{v}$ (thick black line to the left) and $v$ for the whole sample set (to the right) at $x=479$ ( $\mathrm{B}^{\prime}$ in Figs. 5 and 7 ). The line plots and contours (dark and light indicate negative and positive, respectively) are scaled by three times the local rms of the azimuthal velocity $v_{\mathrm{rms}}$, corresponding to (a) $\pm 3 v_{\mathrm{rms}}= \pm 2.4 \times 10^{-1}$ at $z=0.6$; (b) $\pm 3 v_{\mathrm{rms}}= \pm 2.8 \times 10^{-1}$ at $z=1.2$; and (c) $\pm 3 v_{\mathrm{rms}}= \pm 2.0 \times 10^{-1}$ at $z=2.4$. The detected location of the vortex is marked by the red line on the right-hand plot (see $10^{\circ} \lesssim \theta \lesssim 30^{\circ}$ ). 
(a)

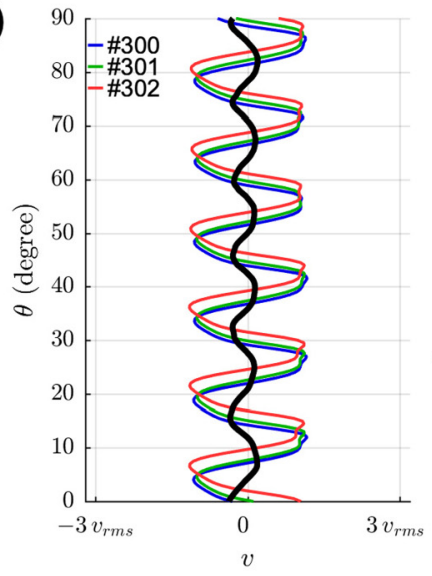

(b)

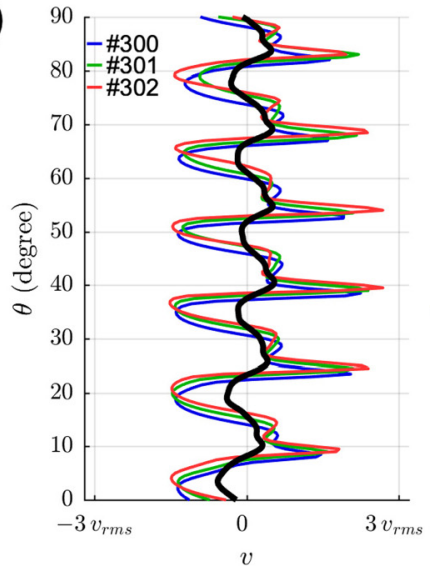

(c)

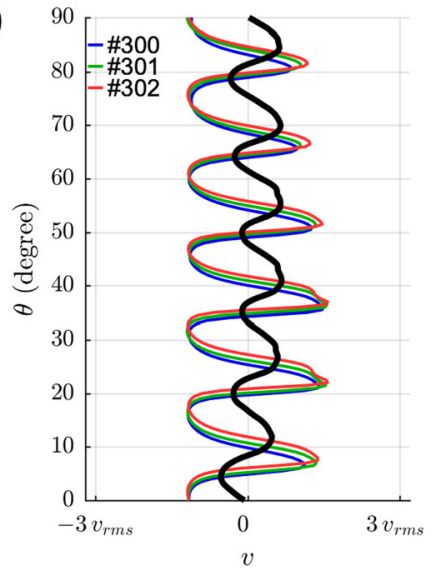

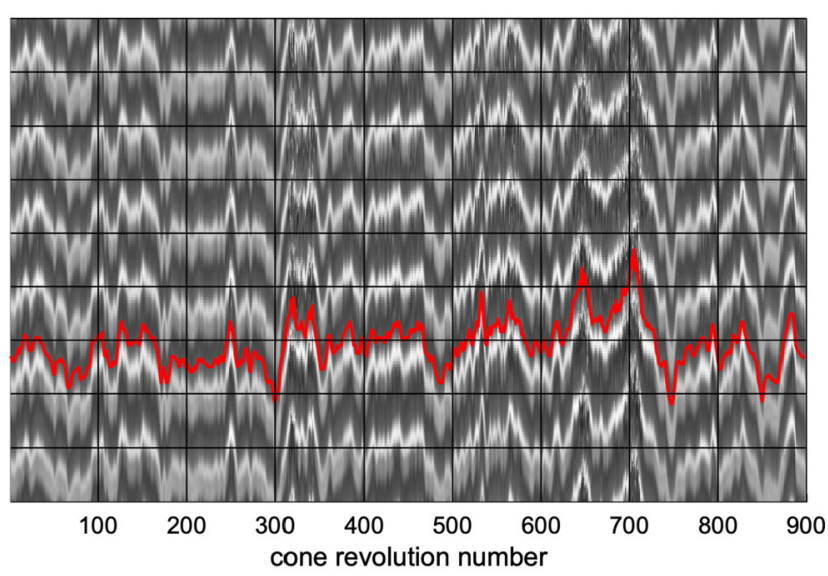
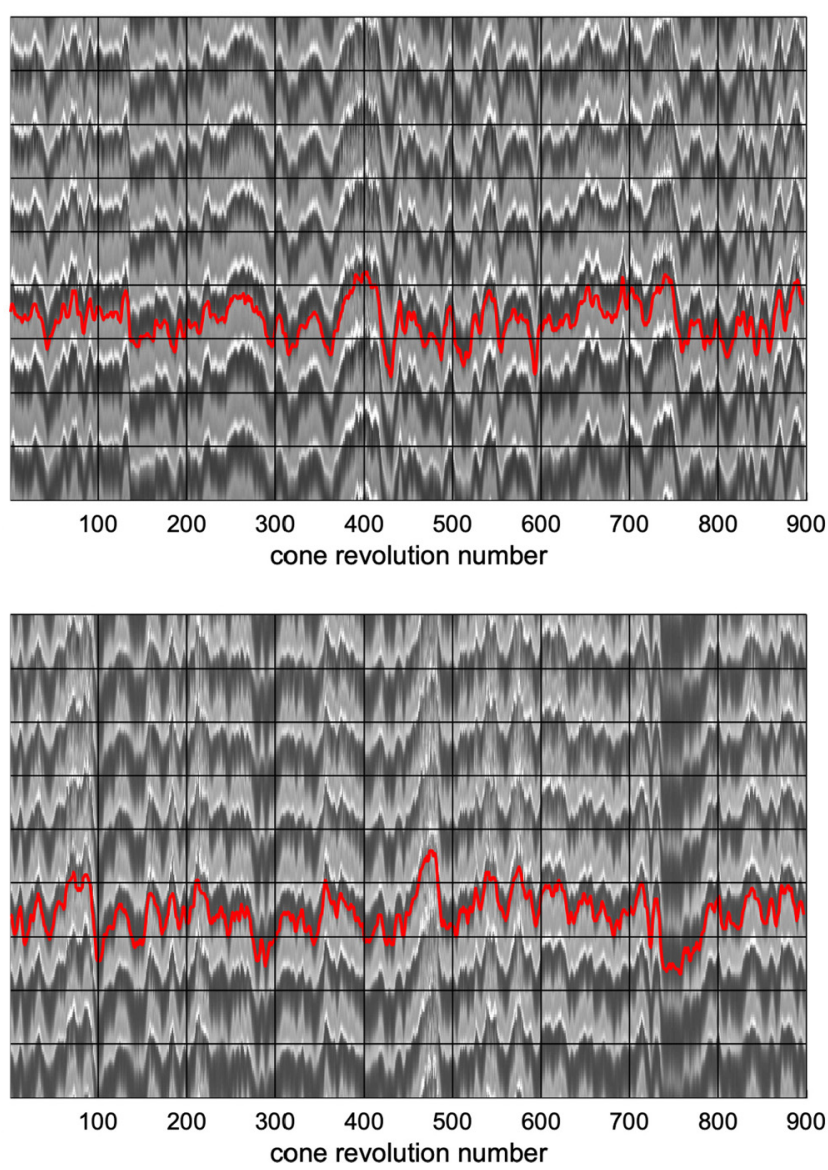

FIG. 9. Velocity fluctuation $v$ for single revolutions (thin colored lines to the left; blue, green, and red lines indicate $v$ for revolution numbers 300,301 , and 302, respectively.), phase-averaged velocity $\tilde{v}$ (thick black line to the left) and $v$ for the whole sample set (to the right) at $x=498$ ( $\mathrm{C}^{\prime}$ in Figs. 5 and 7). The line plots and contours (dark and light indicate negative and positive, respectively) are scaled by three times the local rms of the azimuthal velocity $v_{\mathrm{rms}}$, corresponding to (a) $\pm 3 v_{\mathrm{rms}}= \pm 3.7 \times 10^{-1}$ at $z=1.2$; (b) $\pm 3 v_{\mathrm{rms}}= \pm 2.6 \times 10^{-1}$ at $z=2.4$; and (c) $\pm 3 v_{\mathrm{rms}}= \pm 2.5 \times 10^{-1}$ at $z=3.2$. The detected location of the vortex is marked by the red line on the right-hand plot (see $20^{\circ} \lesssim \theta \lesssim 45^{\circ}$ ). 
(a)

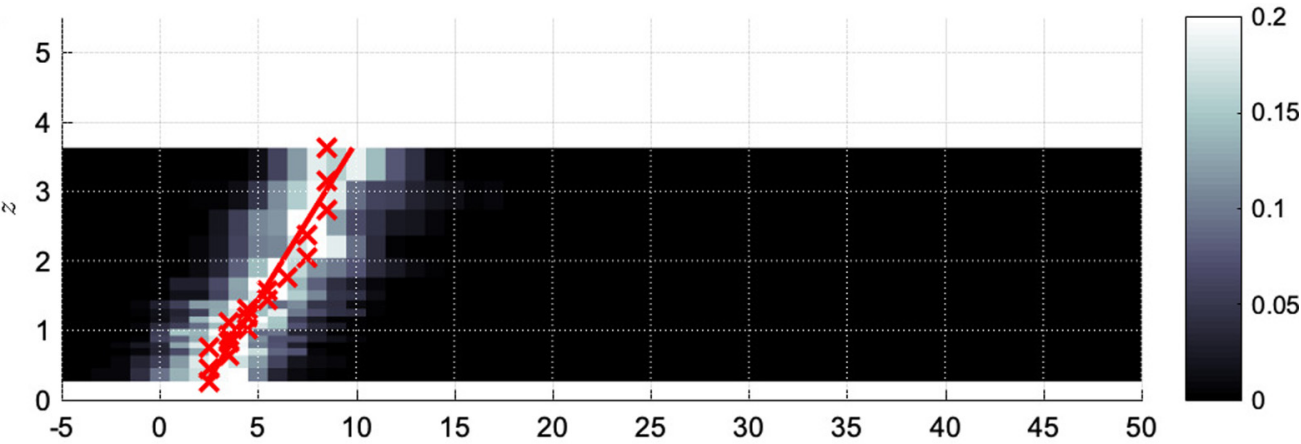

(b)

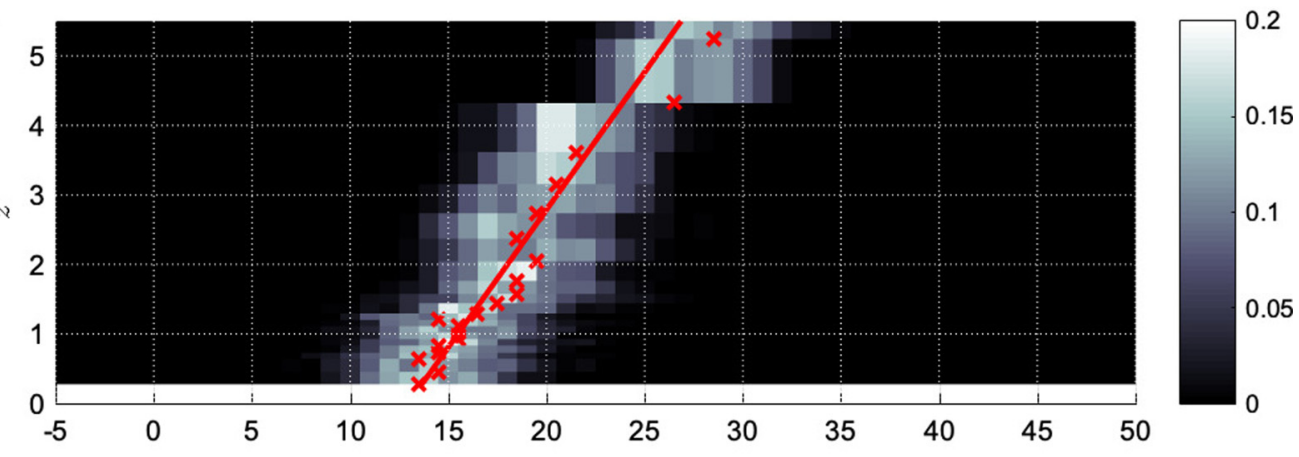

(c)

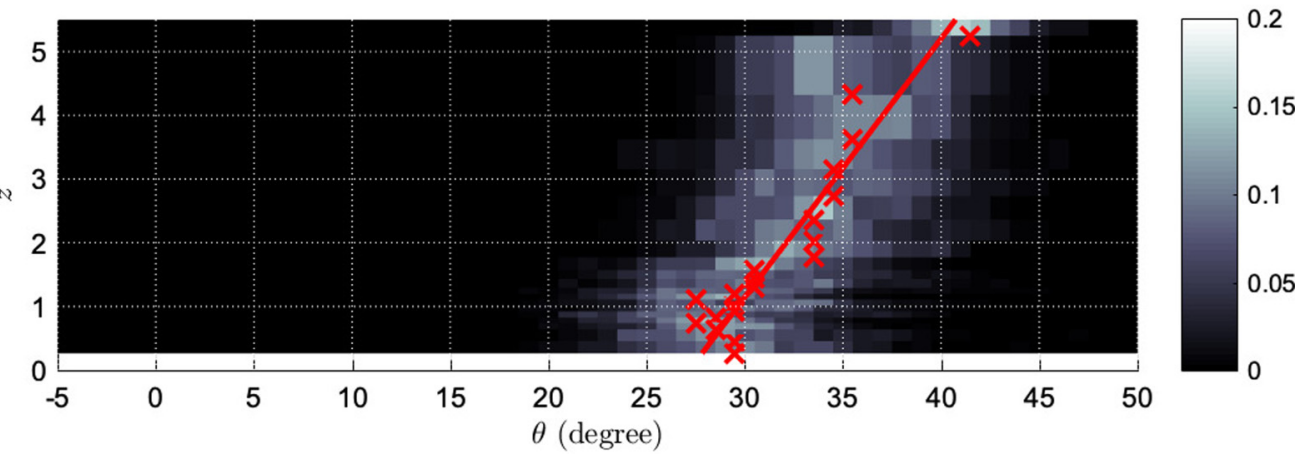

FIG. 10. Probability density function of the azimuthal location of the spiral vortex at (a) $x=461$, (b) $x=$ 479, and (c) $x=498$ : the red cross shows the mean azimuthal locations of the whole population (approx. 900 revolutions) at each wall height. The most probable location of the vortex is determined by a linear fitting of the mean locations (shown by the straight lines). The most probable 100 samples were extracted around the most probable location of the vortex.

measurements. The vortex detection mentioned above was conducted for all $z$ positions. The gray contours in Fig. 10 indicate the probability density function of the detected location of the vortices at different $x$ locations. At each wall height, the mean location is marked by the cross. To reconstruct a smooth "snapshot" of the most probable vortex structure, the most probable location of the vortex is determined by a linear fitting to the mean locations based on the least-square method. The red straight lines in Fig. 10 show the most probable location, leaning toward positive $\theta$ for all $x$ locations. As can be seen the variation around the mean locations increases with $x$. We regarded 100 samples with the vortex located close to the fitting line as the most probable samples and calculated the phase-averaged field from the 100-sample population to give a "snapshot" of the most probable 


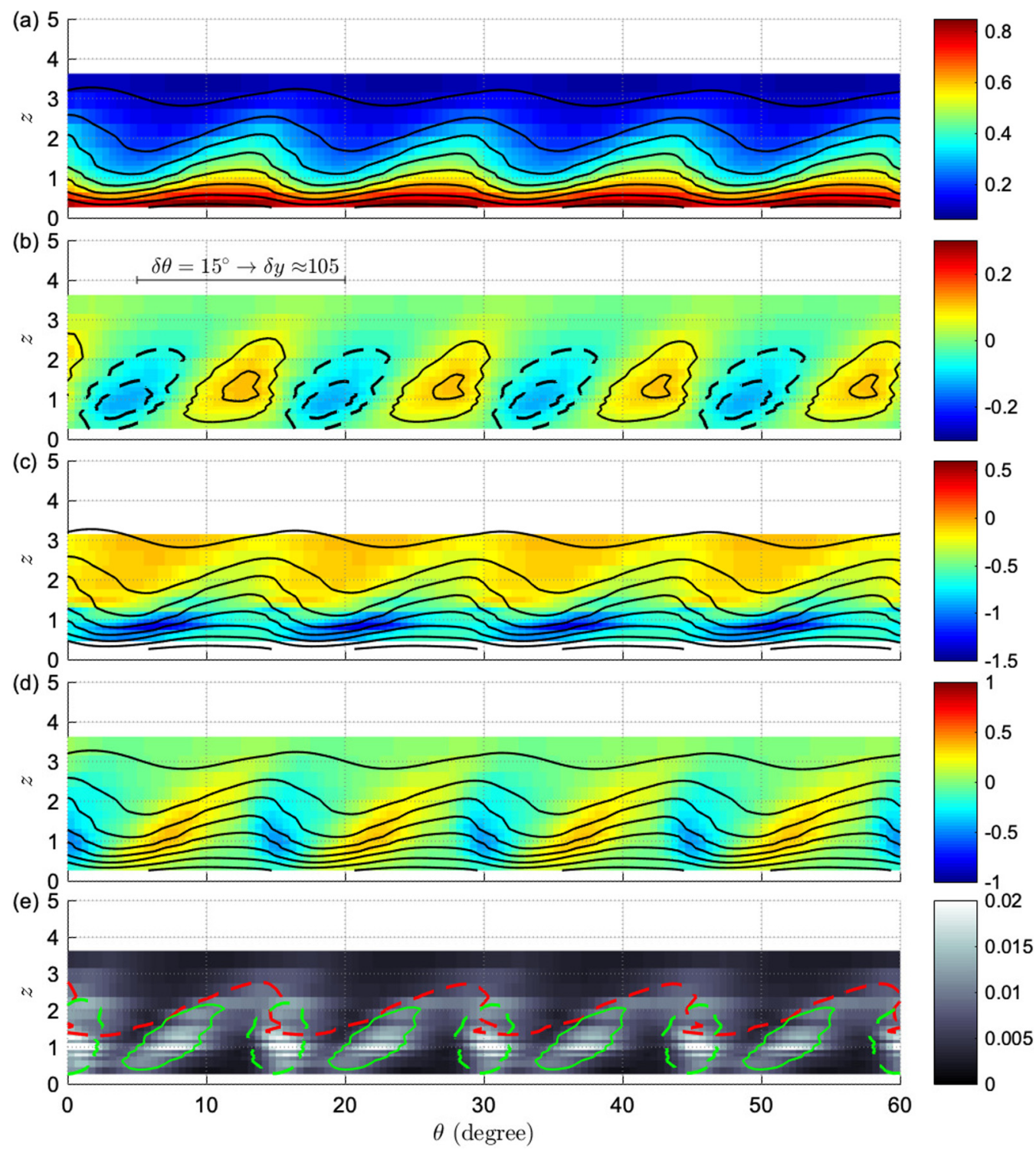

FIG. 11. Reconstructed vortex structures based on the 100-sample population at $x=461$ (A' in Figs. 5 and 7): (a) phase-averaged azimuthal velocity $V+\langle v\rangle$; (b) $\langle v\rangle$; (c) $z$ gradient of the phase-averaged azimuthal velocity $\partial(V+\langle v\rangle) / \partial z$; (d) $\theta$ gradient of the of the phase-averaged azimuthal velocity $\partial\langle v\rangle /(r \partial \theta)$; and (e) rms of the 100 samples. The lines in (a), (c), and (d) are the same and show contours with a spacing of 0.1 . The lines in (b) show contours with a spacing of 0.05 . The green and red contours in (e) indicate isoline of \pm 0.2 in the $z$ and $\theta$ gradients of the mean flow. Negative contours are dashed.

vortex structure. The variation of the detected locations within the 100 samples within 2.0 degrees. Thus, the reconstructed "snapshot" shows a quasi-frozen vortex structure.

Figures 11, 12, and 13 show $\theta z$ planes from the reconstructed snapshots, based on the 100-sample populations discussed above. Three $x$ positions, $x=461,479$, and 498, are shown (corresponding to sections $\mathrm{A}^{\prime}, \mathrm{B}^{\prime}$, and $\mathrm{C}^{\prime}$ in Fig. 5 and Fig. 7). Plot (a) in each figure shows the phase average of the azimuthal velocity field $V+\langle v\rangle$ of the sample population, where $V(z ; x)$ is the mean velocity shown in Fig. 3(b), and $\langle v\rangle$ denotes a phase average of the velocity $v(\theta, z ; x)$ of the 100 -sample population. 

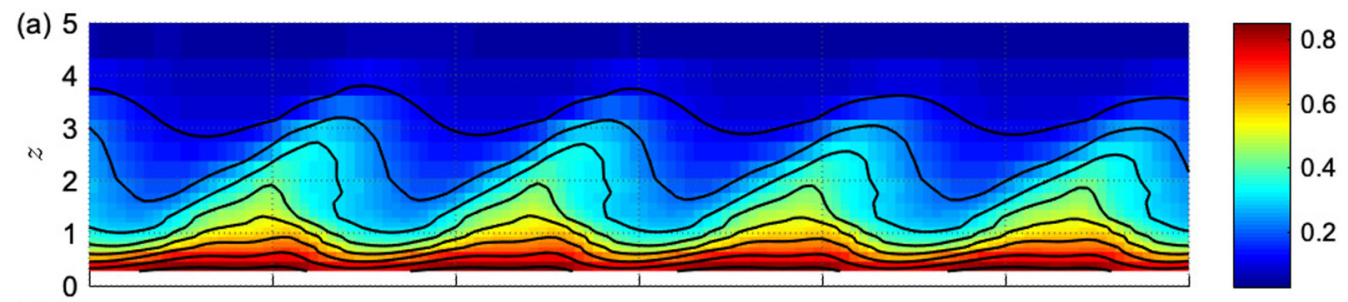

(b)
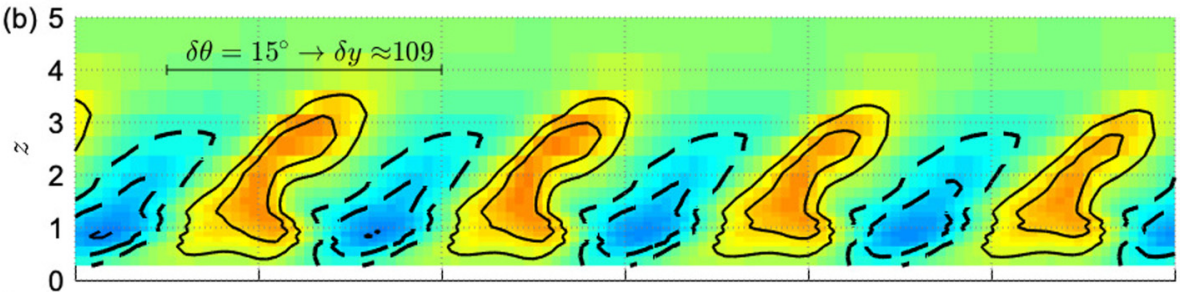

(c)

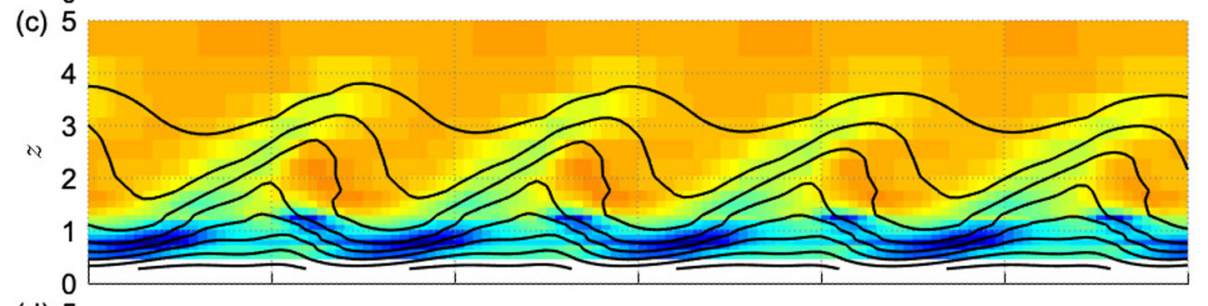

(d)

0
5

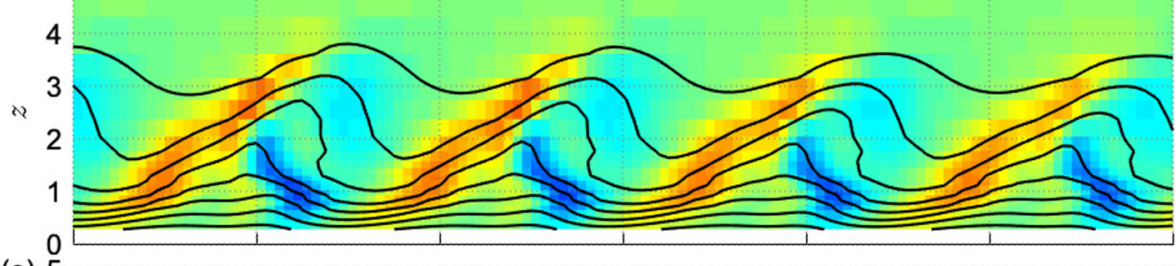

(e)

0
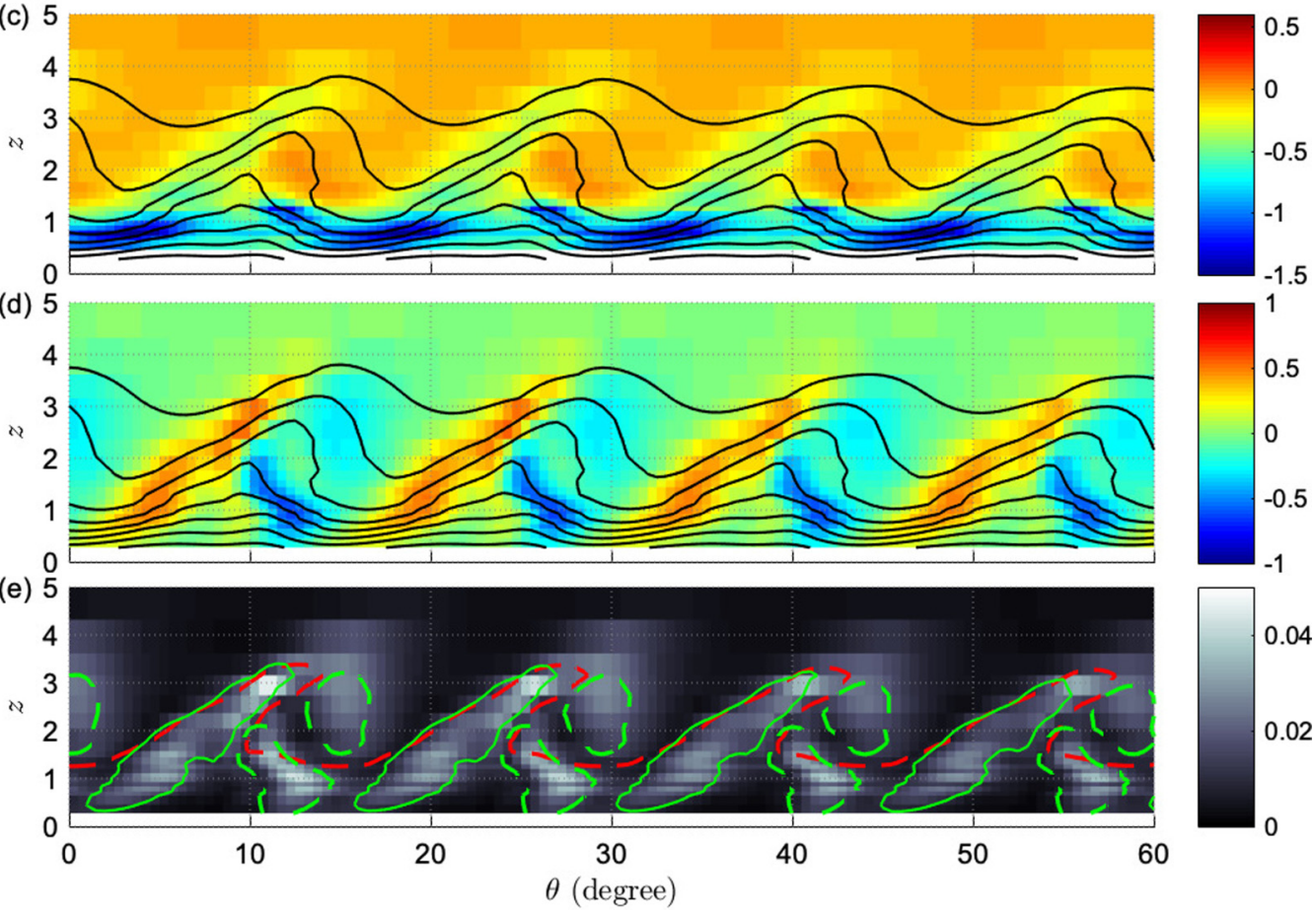

FIG. 12. Reconstructed vortex structures based on the 100-sample population at $x=479$ (B' in Figs. 5 and 7): (a) phase-averaged azimuthal velocity $V+\langle v\rangle$; (b) $\langle v\rangle$; (c) $z$-gradient of the phase-averaged azimuthal velocity $\partial(V+\langle v\rangle) / \partial z$; (d) $\theta$-gradient of the of the phase-averaged azimuthal velocity $\partial\langle v\rangle /(r \partial \theta)$; and (e) rms of the 100 samples. The lines in (a), (c), and (d) are the same and show contours with a spacing of 0.1. The lines in (b) show contours with a spacing of 0.05 . The green and red contours in (e) indicate isoline of \pm 0.2 in the $z$ and $\theta$ gradients of the mean flow. Negative contours are dashed.

The fields are shown in the laboratory frame (hence, $V+\langle v\rangle=1$ at the wall). All three figures show a wavy flow field as expected. Plot (b) in each figure shows $\langle v\rangle$, i.e., the velocity with the mean subtracted, whereas plots (c) and (d) show the spatial gradients of $\partial(V+\langle v\rangle) / \partial z$ and $\partial\langle v\rangle /(r \partial \theta)$, respectively. Finally plot (e) shows the rms of the 100-sample revolutions (plots (c)-(e) will be discussed in the next section). In each figure, only data for one sixth of the cone $\left(0^{\circ} \leqslant \theta \leqslant 60^{\circ}\right.$, covering four vortices) are shown. As discussed in relation to Fig. 5, the disturbances introduced 


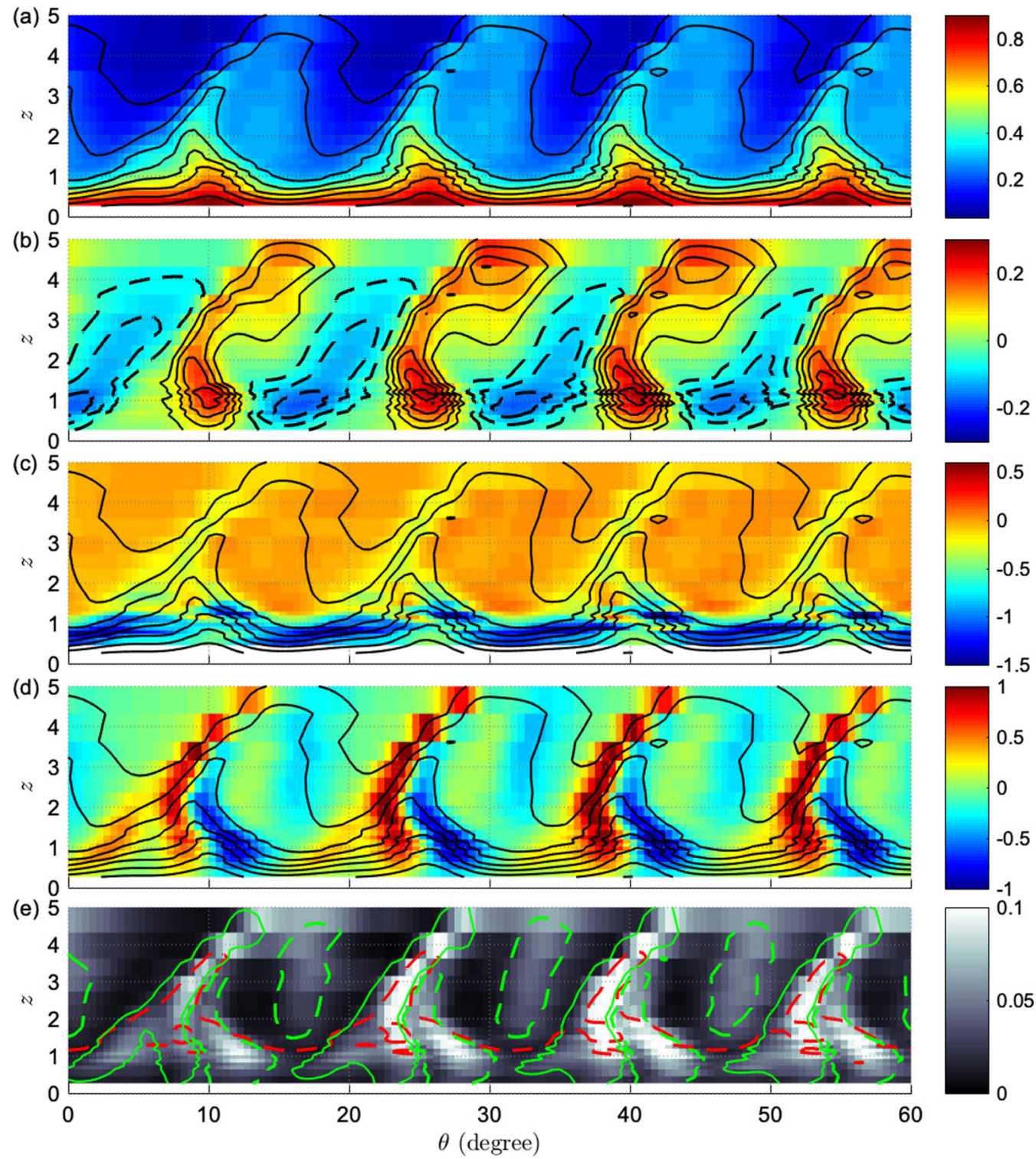

FIG. 13. Reconstructed vortex structures based on the 100 -sample population at $x=498$ (C' in Figs. 5 and 7): (a) phase-averaged azimuthal velocity $V+\langle v\rangle$; (b) $\langle v\rangle$; (c) $z$ gradient of the phase-averaged azimuthal velocity $\partial(V+\langle v\rangle) / \partial z$; (d) $\theta$ gradient of the phase-averaged azimuthal velocity $\partial\langle v\rangle /(r \partial \theta)$; and (e) rms of the 100 samples. The lines in (a), (c), and (d) are the same and show contours with a spacing of 0.1 . The lines in (b) show contours with a spacing of 0.05 . The green and red contours in (e) indicate isoline of \pm 0.2 in the $z$ and $\theta$ gradients of the mean flow. Negative contours are dashed.

by the artificial roughnesses develop uniformly although the variation among the vortices increases downstream.

Figures 11(b), 12(b), and 13(b) provide a clearer explanation of the $z$ structure shown in Figs. 7( $\left.\mathrm{A}^{\prime}\right), 7\left(\mathrm{~B}^{\prime}\right)$, and $\left.7 \mathrm{C}^{\prime}\right)$. In Fig. 11(b), positive (negative) $\langle v\rangle$ occupies the wider area in $z \lesssim 1.2$ $(z \gtrsim 1.2)$, corresponding to the maximum in the positive (negative) $v$ in Fig. 7( $\left.\mathrm{A}^{\prime}\right)$. A similar inclined structure is also observed in DNS for the disk case (Fig. 11 in Ref. [24]). In Fig. 12(b), 
the high-momentum upwelling region begins to be distorted and a spacing appears between highand low-momentum regions in $1.2 \lesssim z \lesssim 1.5\left(12^{\circ} \lesssim \theta \lesssim 15^{\circ}, 27^{\circ} \lesssim \theta \lesssim 30^{\circ}\right.$, and $41^{\circ} \lesssim \theta \lesssim 45^{\circ}$ ), corresponding to the branched maximum in Fig. 7( $\left.\mathrm{B}^{\prime}\right)$. Further away from the wall, the branched maximum in the PDF indicates the top part of the high-momentum upwelling. In Fig. 13(b), the distortion and spacing become more significant and the branched maximum extends toward the outer layer in Fig. 7( $\left.\mathrm{C}^{\prime}\right)$. Thus, the branched maximum in Figs. $7\left(\mathrm{~B}^{\prime}\right)$ and $7\left(\mathrm{C}^{\prime}\right)$ indicates the distortion of the high-momentum area, or overturning process of the high-momentum upwelling.

The detailed overturning process discussed above is not observable in the phase-averaged velocity field $\tilde{v}$. As observed in Figs. 8 and 9, the vortex meandering causes phase-shifting of the waveforms and each plateau is smoothed out in the phase-averaged velocity. However, the PDF extracts only the value of $v$ without the phase and is, therefore, unaffected by the phase-shifts. Thus, the PDF detects the overturning process from the point measurement without any complicated vortex-location detection. The PDF can probably also detect the overturning process (in a timeaveraged sense) on the clean cone [see the similar branched maxima in Figs. $7(\mathrm{C})$ and $7\left(\mathrm{C}^{\prime}\right)$ ], even when vortex splitting and merging occur and the reconstruction of the snapshot becomes more difficult. This is clearly a distinct advantage of the PDF method.

\section{Secondary instability and vortex meandering}

To investigate the secondary instability, we evaluate the mean-flow distortion. In Figs. 11(c), $12(\mathrm{c})$, and $13(\mathrm{c})$, the stronger shear in the $z$ direction appears in the near-wall region $(z \leqslant 1.5)$, where the low-momentum downwelling exists, while the shear becomes weaker but extends toward the far-wall region on the high-momentum upwelling. In plot (d) of each figure, the larger magnitude of (negative and positive) $\theta$ gradients can be observed between the high- and low-momentum areas. As $x$ increases, the areas become more asymmetric and the positive $\theta$ gradient extends to the outer layer more significantly. These areas having a large magnitude of $z$ and $\theta$ gradients are marked by the isolines of \pm 0.2 in each plot (e).

The gray contours in Figs. 11(e), 12(e), and 13(e) show the rms of the most probable 100-sample revolutions, indicating that the areas having the high rms overlap with the areas with large spatial gradients. We also conducted this evaluation with different numbers of samples, e.g., 20 and 50 samples although only results using 100 samples are shown here. We found the same overlapping structures regardless of the number of samples. These results indicate that the rms is mainly caused by nonstationary disturbances rather than the slight shift of the meandering vortices within the sample population. A similar structure is also observed around the breakdown in swept-wing boundary layers (e.g., Fig. 28 in Ref. [10]).

Another point of interest is the relation between the secondary instability and vortex meandering. Figure 14 shows the "sorted" azimuthal velocity fluctuation $v$ at $x=498$ for three different $z$; the same data as Fig. 9 are shown but all the revolutions are sorted according to the detected vortex location, which is marked by the solid white curve. As can be seen for all three $z$ positions all vortices are in phase across the entire cone. The data on the left side of the figures correspond to the revolutions in which the vortices are located upstream (smaller $\theta$ ). Figure 14 indicates that irregular oscillations appear more significantly on the right side of the figure (data where the vortices are located downstream) than on the left side. These oscillations were not clearly observed at $x=461$ and 479; Taking a close look at Fig. 9, one can see the irregular oscillations, e.g., around the 700th revolution in Fig. 9(a) but not in Fig. 8.

For a clearer visualization of this trend, the velocity fluctuation $v$ at some single revolutions marked by the dashed lines in Fig. 14 is plotted as curves in Fig. 15; the sorted $v$ for the 20th, 150th, 300th, 600th, 750th, and 870th revolutions corresponds to the curves from the bottom to the top of each subplot in Fig. 15. Note that the most probable 100 samples that were used for the reconstruction of the "snapshot" in the previous section correspond to data between two squares in Fig. 14 and are located in the range between the 300th and 600th revolutions. Figure 15 clearly indicates that $v$ begins to contain high-frequency oscillations at higher values of the sorted cone 

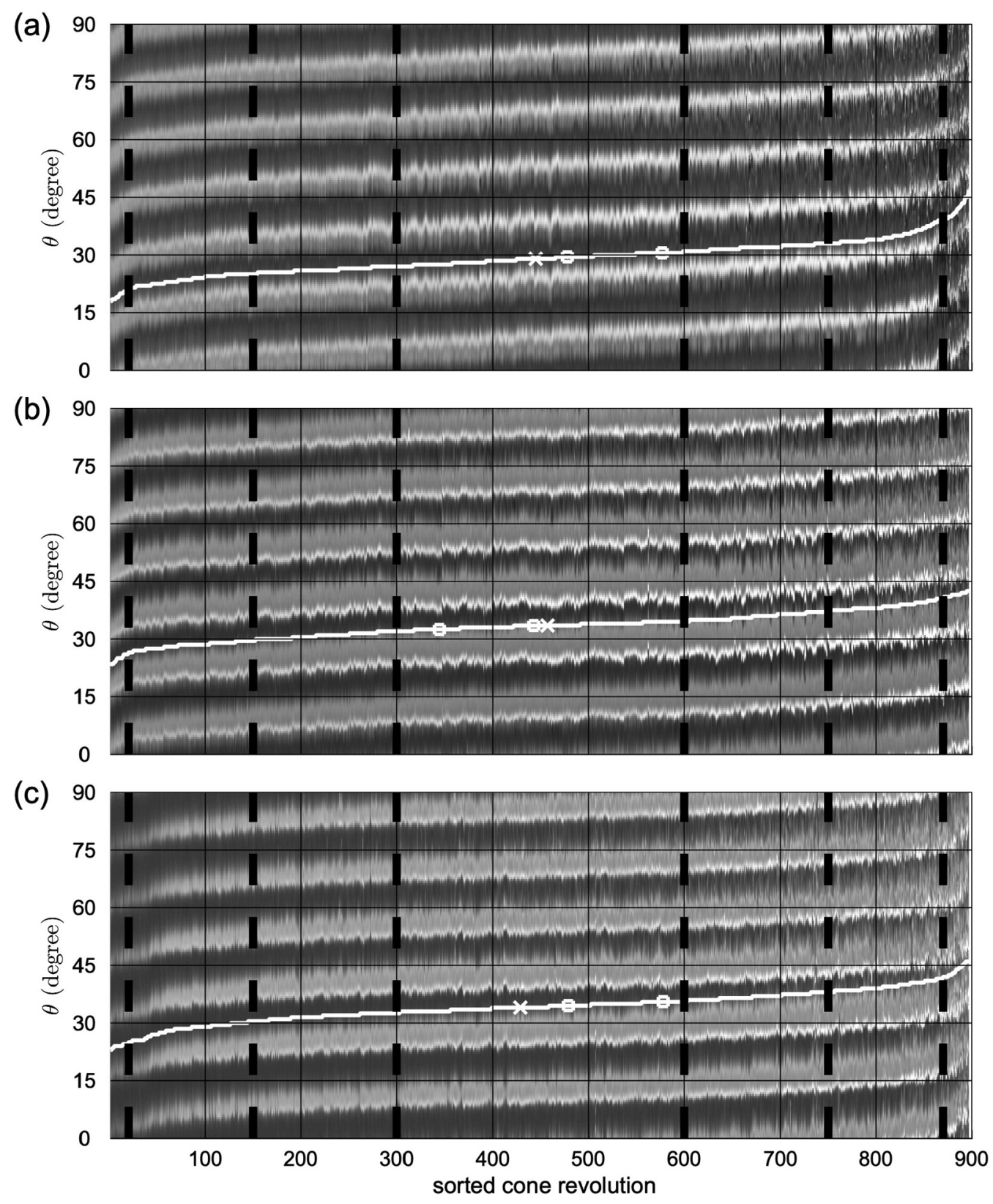

FIG. 14. Velocity fluctuation $v$ (gray contour) sorted by the detected vortex location (shown by the solid curve) at $x=498$ ( $\mathrm{C}^{\prime}$ in Figs. 5 and 7). The cross shows the mean location of the vortex at each wall height: (a) $z=1.2$, (b) $z=2.4$, and (c) $z=3.2$. The data between two squares were used for the reconstruction. The contours are scaled in the same way as in Fig. 9. The dashed lines indicate the data shown in Fig. 15.

revolution, corresponding to that the vortex is shifted downstream (larger $\theta$ in Fig. 14), at all wall heights. Especially, data for the 750th and 870th revolutions contain waves, with a wavelength much shorter than the one of the primary vortex $\left(15^{\circ}\right.$ in $\left.\theta\right)$. It should be mentioned that the waveforms for the 750th and 870th revolutions are similar to the ones shown in Figs. 18(b) and 18(c) in Ref. [16]. 
(a)

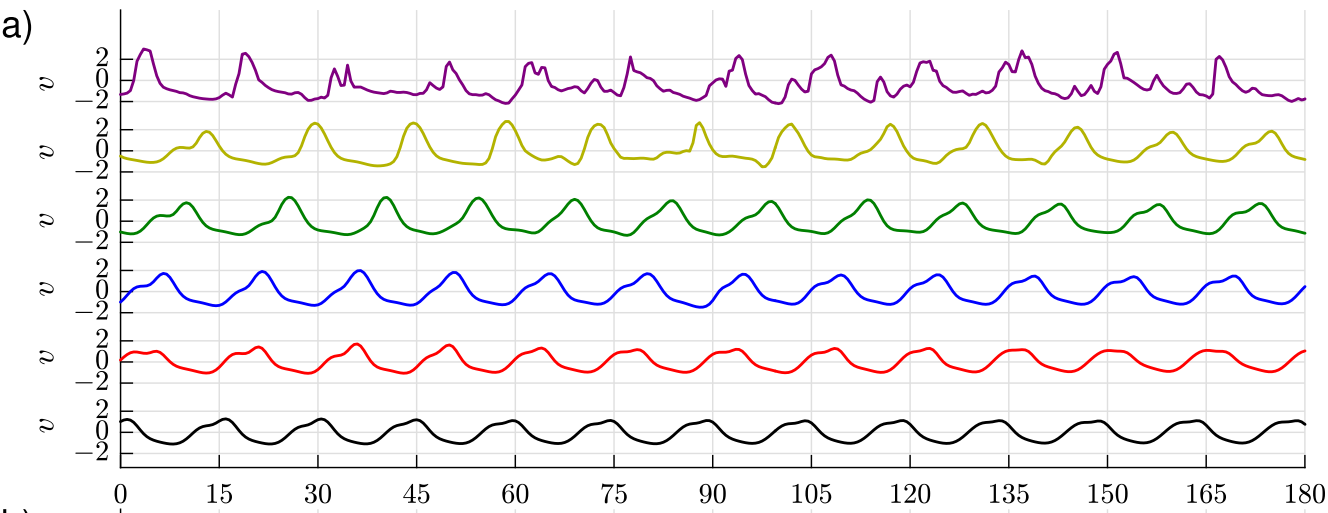

(b)

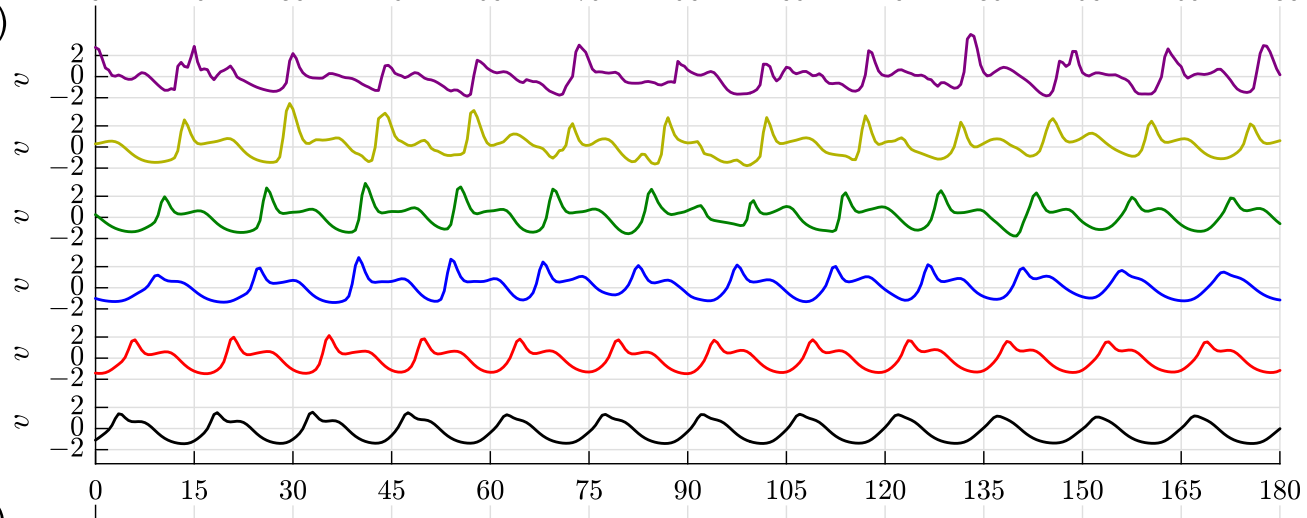

(c)

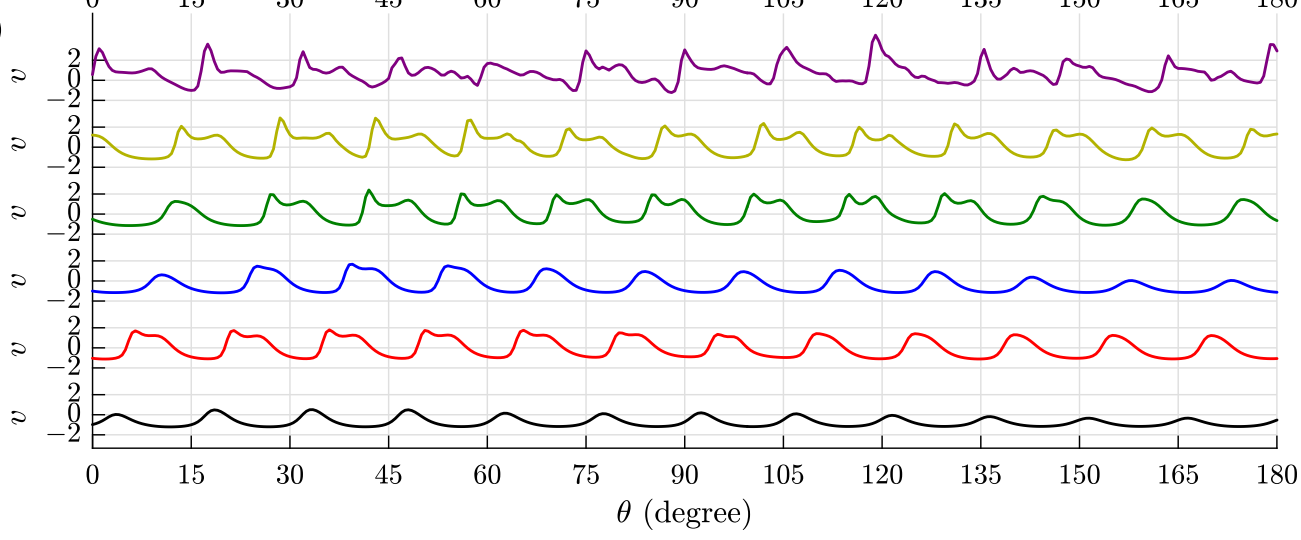

FIG. 15. Velocity fluctuation $v$ at $x=498$ ( $\mathrm{C}^{\prime}$ in Figs. 5 and 7) on the cone with roughnesses for different vortex locations during the vortex meandering: the curves from the bottom to top in each figure show the velocity fluctuation $v$ for the 20th, 150th, 300th, 600th, 750th, and 870th revolutions of the sorted data, corresponding to the data marked by the dashed lines in Fig. 14. The plots are scaled by twice the local rms of the azimuthal velocity $v_{\text {rms }}$, corresponding to (a) $\pm 2 v_{\text {rms }}= \pm 2.5 \times 10^{-1}$ at $z=1.2$; (b) $\pm 2 v_{\text {rms }}= \pm 1.7 \times 10^{-1}$ at $z=2.4$; and (c) $\pm 2 v_{\mathrm{rms}}= \pm 1.7 \times 10^{-1}$ at $z=3.2$.

A similar evaluation was also made for the clean cone data and the velocity fluctuation $v$ for the typical single revolutions when the vortex is located (relative to the mean vortex position) upstream (bottom), intermediate (middle), and downstream (top) are shown in Fig. 16. Here, the data are extracted from the locations marked by the dashed lines in Fig. 22. Because the vortices are splitting 
(a)

(b)
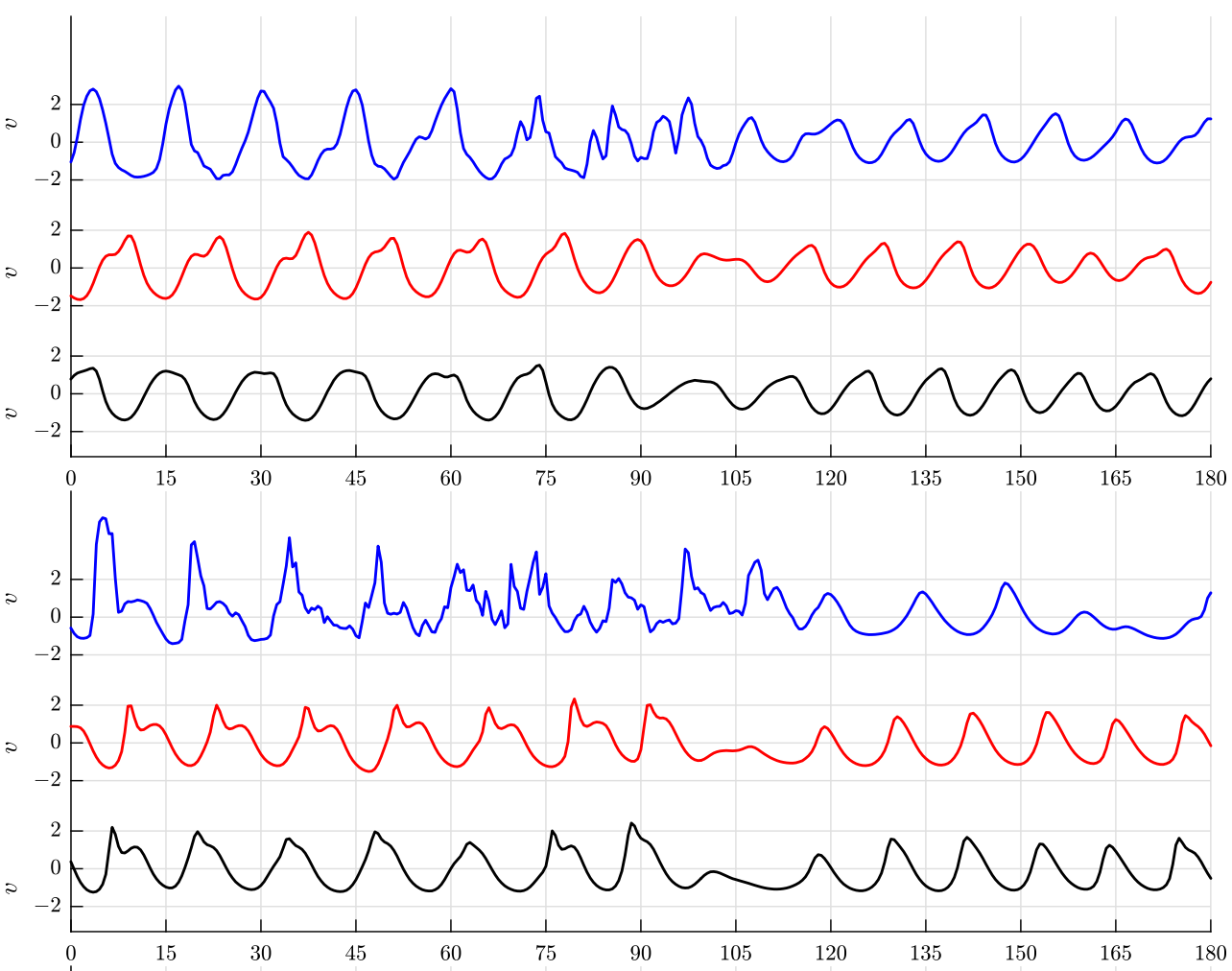

(c)

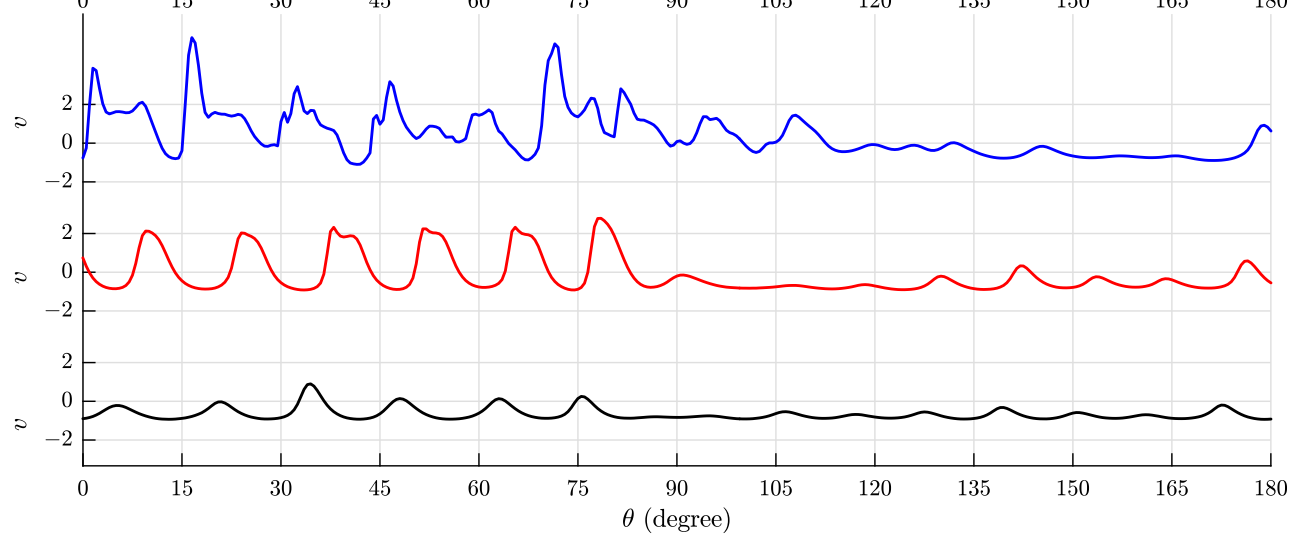

FIG. 16. Velocity fluctuation $v$ for typical single revolutions when the vortex is shifted (relative to the mean vortex position) upstream (bottom), intermediate (middle), and downstream (top). All data correspond to those marked by the dashed lines in Fig. 22 ( $x=535$ on the clean cone, C in Figs. 5 and 7). Each plot is scaled by twice the local rms of the azimuthal velocity $v_{\mathrm{rms}}$, corresponding to (a) $\pm 2 v_{\mathrm{rms}}= \pm 2.2 \times 10^{-1}$ at $z=1.2$; (a) $\pm 2 v_{\mathrm{rms}}= \pm 1.6 \times 10^{-1}$ at $z=2.4$; and (a) $\pm 2 v_{\mathrm{rms}}= \pm 1.4 \times 10^{-1}$ at $z=3.2$.

and merging (see Appendix B), the vortex location cannot be detected by the minimum-searching algorithm and the data were selected manually.

Figure 16 shows that the amplitudes of the vortices in the clean cone case are quite different depending on the azimuthal location because in this case the vortices are triggered by nonuniform, random surface roughnesses. In contrast, the signal on the cone with the artificial roughness elements is very uniform at least when the vortex is located upstream as shown in the lower lines 


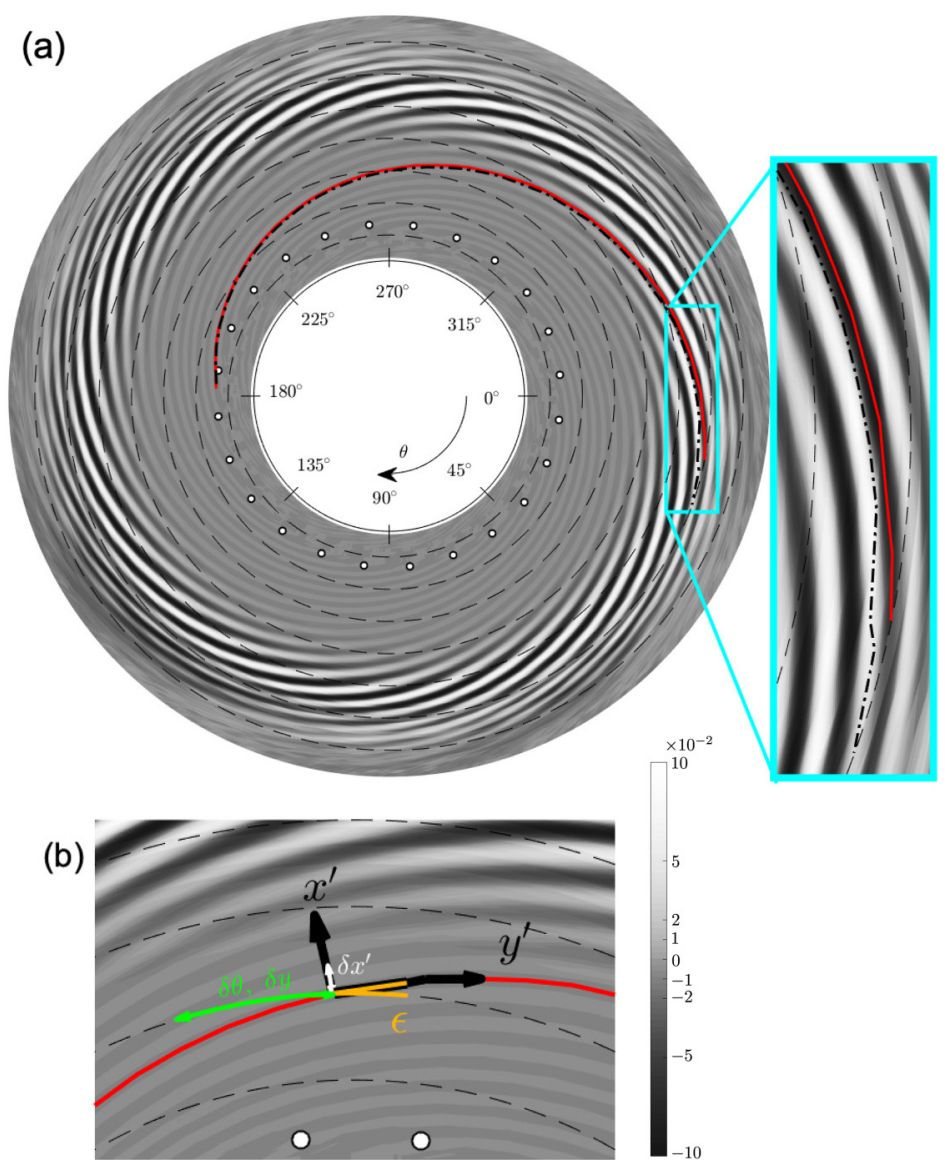

FIG. 17. Phase-averaged velocity $\tilde{v}$ on the cone with roughnesses at $z=1.2$ : The white dots are at $x=267$ and show the location of the roughnesses. The solid and dash-dotted lines mark the detected most probable vortex location and shifted location downstream based on the tracking of the local minimum of averaged $v$ for the 100-sample population. The dashed lines indicate the locations at $x=250,300,350,400,450,500$, and 550. (b) Blow-up of (a) with the orthogonal curvilinear coordinate system $\left(x^{\prime}, y^{\prime}\right)$ fixed on the vortex. The angle $\epsilon$ is the angle of the vortex ( $y^{\prime}$ axis) with respect to the azimuthal direction.

in Fig. 15. In Fig. 16, the disturbances with different amplitudes on the clean cone indicate an amplitude dependency; the high-frequency oscillations appear on the larger vortices $\left(\theta \lesssim 110^{\circ}\right)$ when the vortices are shifted downstream (top line in each subplot) but not on the smaller vortices $\left(\theta \gtrsim 120^{\circ}\right)$. This may be consistent with the two transition scenarios shown by the numerical simulations on the disk [16], where the secondary instability was observed in a wider area for the case with a larger stationary forcing.

To characterize the effects of the vortex meandering on the spiral vortex structure, we also consider the aspect ratio of the vortex cross section. Figure 17(a) shows the phase-averaged velocity field $\tilde{v}$ at $z=1.2$ on the cone with artificial roughnesses in a gray contour scale. The solid and dash-dotted lines indicate the mean vortex locations detected by searching for the minimum of $v$ for the 100 samples around the mean vortex location and for the 100 samples when the vortex are located farthest downstream (the last 100 revolutions in Fig. 14(a), for example. For each case, no fitting was applied for this case). Here, we define a new orthogonal curvilinear coordinate system $\left(x^{\prime}, y^{\prime}, z^{\prime}\right)$ on the detected vortex locations as shown in Fig. 17(b). The $x^{\prime}$ and $y^{\prime}$ axes are normal and parallel to the vortex axes. The $z^{\prime}$ axis is normal to the cone surface (same as $z$ ). The angle $\epsilon$ 

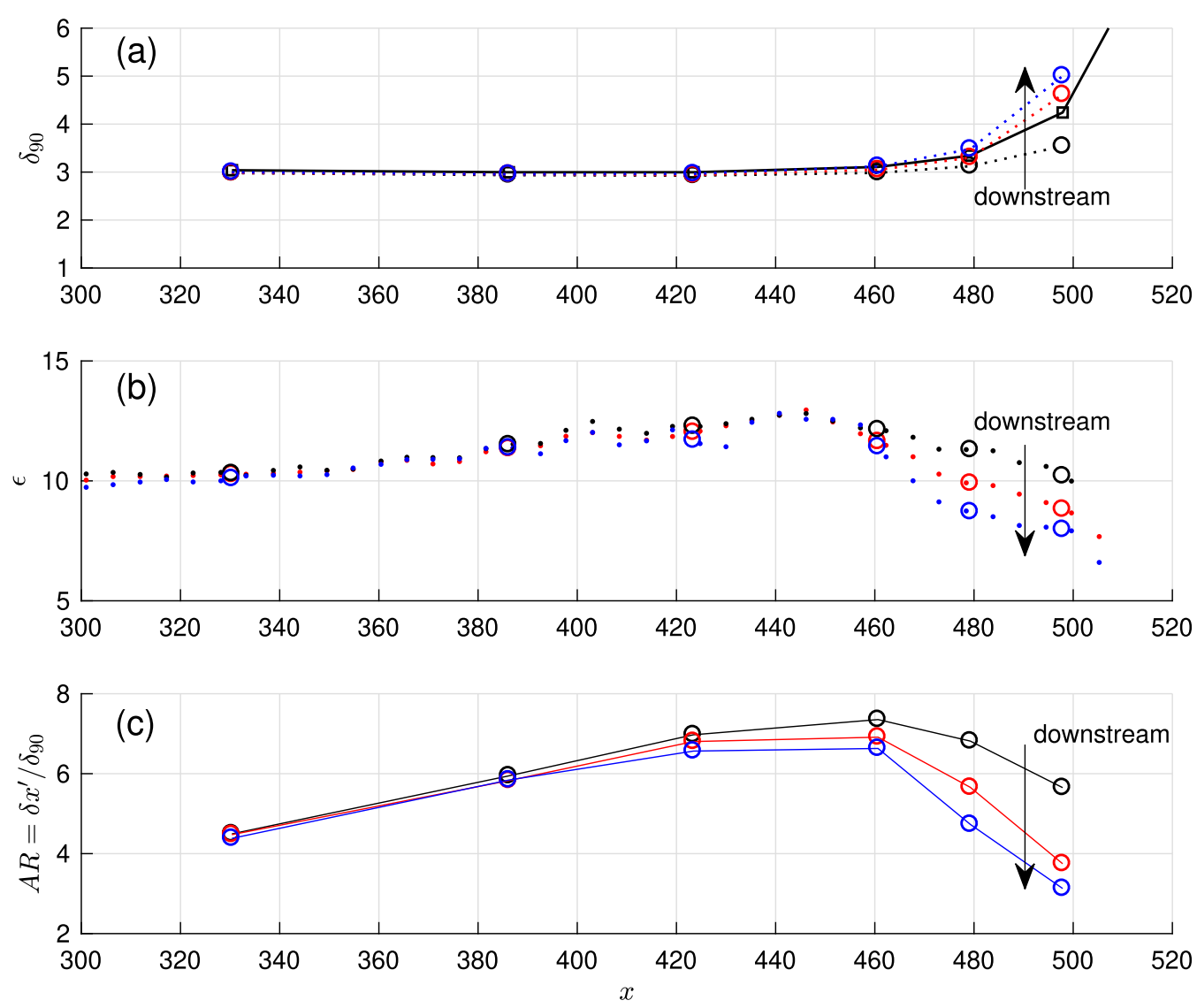

FIG. 18. Effect of the vortex meandering on the $90 \%$ boundary-layer thickness and spiral vortices as a function of $x$ : (a) $90 \%$ boundary-layer thickness $\delta_{90}$, (b) angle of the vortex $\epsilon$, and (c) aspect ratio $A R$ of the vortex on the $x^{\prime} z^{\prime}$ plain. The black, red, and blue markers show quantities calculated by data for 100 revolutions, at which the vortex is located upstream of, intermediate to, and downstream of the mean, respectively. In (b), the dots correspond to the angle calculated based on the detected vortex axis (shown in Fig. 17) at $z=1.2$. The circles are interpolated angles at the location where the profile measurements were conducted. The squares in (a) show the time-averaged $90 \%$ boundary-layer thickness, shown in Fig. 3(b) as a reference. Sections at $x=461,479,498$ are denoted by $\mathrm{A}^{\prime}, \mathrm{B}^{\prime}, \mathrm{C}^{\prime}$, in Figs. 5 and 7.

is defined as the angle of the $y^{\prime}$-axis with respect to the azimuthal direction. We estimate the aspect ratio of the spiral vortex in the $x^{\prime} z^{\prime}$ plane, $A R=\delta x^{\prime} / \delta_{90}$, where the width of the vortex is defined as $\delta x^{\prime}=(2 \pi x \sin \psi \sin \epsilon) / 24$. The vortex height is estimated as the $90 \%$ boundary-layer thickness $\delta_{90}$. It should be noted that $\delta_{90}$ is a rough measure of the vortex height and tends to underestimate the vortex height in the transition region (see Fig. 13: the high-momentum upwell is located clearly beyond the $90 \%$ boundary-layer thickness, $\delta_{90}=4.6$.).

The effects of the vortex meandering on the vortex aspect ratio as a function of $x$ are shown in Fig. 18. In this figure we divide the vortices into three categories (with 100 revolutions each) depending on their azimuthal location relative to the mean vortex position at each $x$ and $z$ position: those located upstream (having the vortex axis at smaller $\theta$, i.e., the first 100 revolutions in Fig. 14, for example) are marked by black symbols; those located downstream (having the vortex axis at larger $\theta$, i.e., the last 100 revolutions in Fig. 14) are marked by blue symbols; and those located around the mean are here called "intermediate" (100 revolutions around the average) marked by red 
symbols. Figure 18(a) shows the $90 \%$ boundary-layer thickness $\delta_{90}$ for the three cases as well as the time-averaged thickness, whereas Fig. 18(b) shows the angle $\epsilon$ of the vortex with respect to the azimuthal direction as shown in Fig. 17(b). In Fig. 18(c) the aspect ratio $A R$ of the vortex in the $x^{\prime} z^{\prime}$ plane is given. As a general trend for all vortex locations, $A R$ increases almost linearly with $x$ in the laminar region $(x \leqslant 423)$. In the transition region $(x \geqslant 461), A R$ decreases with $x$ because $\delta_{90}$ increases and $\epsilon$ decreases. Beyond $x=498$, namely, in stage (iii) of Fig. 5(c), the vortices begin to collapse and the location of the vortex cannot be detected. The effect of the vortex meandering seems to resemble a shift in the $x$ location. As the vortex shifts downstream, $\delta_{90}$ increases, $\epsilon$ decreases, and $A R$ decreases.

The effect of the vortex meandering on the aspect ratio of the spiral vortex might be explained by the propagation length of the disturbance from the roughnesses. As shown in Fig. 17(a), the vortex that is shifted downstream has a slightly longer propagation length (larger $y^{\prime}$ ) at a given value of $x$. Therefore, the vortex develops further during a longer propagation length and the aspect ratio becomes smaller and probably the vortex becomes more sensitive to high-frequency oscillations, leading to its collapse.

There are still some ambiguities in this analysis. First, the cause of vortex meandering is not clear. Secondly, we do not know whether or not there is a causal relation between the vortex meandering and the appearance of the secondary instability. Are the high-frequency oscillations observed occasionally where the disturbance has traveled a longer distance and developed further? Or, is there a mechanism by which the secondary instability causes the meandering or is it that the meandering causes the secondary instability? It should be noted that high-frequency oscillations due to the secondary instability and collapse of the spiral vortex are hardly distinguishable in a point measurement in the laboratory frame. To conclude these issues, further investigations are required, e.g., with numerical simulations containing the vortex meandering or experimental data captured in the rotating frame.

In summary, our measurement captured high-frequency oscillations, which may be related to a secondary instability. The high-frequency components appeared at the late stage of overturning of high-momentum upwelling, especially where the vortex is located downstream of the mean vortex position during vortex meandering. Also, disturbances introduced by the nonuniform roughnesses on the clean cone show amplitude dependency; the high-frequency oscillations appear on the vortices with the larger amplitude. However, the cause of vortex meandering and the influence of and on the secondary instability are not clear.

\section{CONCLUSIONS}

The present work reports on the unstable region and the transition process of the boundarylayer flow on a rotating cone with a half angle of 60 degrees using the PDF method introduced by Imayama et al. [20]. The PDF of the azimuthal velocity fluctuation $v$ shows the transition process in a consistent manner both on the rotating disk and on the rotating cone and in both cases with and without artificial roughness elements (Fig. 5). For the early development stage of the spiral cross-flow vortices, the PDF shows the difference due to the uniformity of the initial disturbances; in the case with artificial roughness elements mounted uniformly in the azimuthal direction, the PDF has two maxima corresponding to positive and negative values of $v$. On the clean cone and disk, the PDF has only one maximum around $v=0$ because of the amplitude modulation of the $v$ signal. In the transition stage, however, the PDFs show a similar pattern for both the disk and cone, with and without the roughness elements.

In addition to the PDF, the velocity fluctuations were carefully examined and the most probable vortex structure was reconstructed from the time signals measured by a single point hot wire (Figs. 11, 12, and 13). The results show that the PDF detects the overturning process of the high-momentum upwelling of the spiral vortices (Fig. 7). This overturning process cannot be detected in the phase-averaged velocity because of the meandering of the spiral vortices. Thus, the PDF method has a distinct advantage when assessing the structural nature and development 
of the cross-flow vortices from single-point measurements. The PDF method easily detects the overturning process without any simultaneous plane measurement such as PIV or complicated vortex reconstruction (which was undertaken in this work to verify the method).

Also, our measurement captured the high-frequency oscillations, which may be related to the secondary instability, in the velocity fluctuation. The high-frequency oscillations appeared at the late stage of overturning of the vortices during the transition process ( $C$ and $C^{\prime}$ in Fig. 5 or Fig. 7). They appear particularly on vortices with a larger amplitude when the spiral vortices shift downstream (relative to the mean vortex position) during the vortex meandering (Figs. 14, 15, and 16).

\section{ACKNOWLEDGMENTS}

We thank Dr Shintaro Imayama for providing the data from experiments on the rotating disk and used here for comparison purposes. This work was supported mainly by the Swedish Research Council (D0578601) through the ASTRID project, supporting the consecutive postdoc positions held by the two first authors. Dr Takuya Kawata was also sponsored by a Grant-in-Aid for research fellows by the Japan Society for the Promotion of Science (JSPS, No. 17J04115) for his stay in Stockholm during the writing of this paper. Professor Henrik Alfredsson extends his thanks to Professor Masaharu Matsubara of Shinshu University and the JSPS invitational fellowship for research in Japan program (No. S18061) for supporting his visits to Nagano, where part of this paper was finalized.

\section{APPENDIX A: HOT-WIRE CALIBRATION AND WALL-POSITION DETERMINATION METHODS}

In this section the hot-wire calibration method as well as the determination of the hot-wire position relative to the wall are described. In the present work, the calibration of the hot wire was done in situ in the laminar boundary layer, where the similarity solution on a rotating cone is known (see, for instance, Ref. [9]).

The calibration procedure is based on the velocity distribution within the boundary layer being known as function of the wall-normal coordinate and the wall speed. If the wall distance were known accurately, then it would be easy to find the calibration curve, but in the present case the absolute distance to the wall was not known accurately enough. However, by taking calibration points at different rotational speeds at several fixed wall distances, where the increments between the various wall distances were accurately known, it was possible to estimate the position of the wall relative to the hot-wire sensor through an error minimization procedure. Such a data set of calibration points is shown in Fig. 19 for five different rotational speeds and six different wall-normal positions.

The velocity at the position of the hot wire is given as

$$
V^{*}=\Omega^{*} x^{*} \sin \psi f\left[\left(z_{m}^{*}+z_{0}^{*}\right) / \delta_{v}^{*}\right],
$$

where $z_{m}^{*}$ is the indicated wall distance of the traversing system (adjusted using the optical camera technique) and $z_{0}^{*}$ is the unknown error in the wall distance. Here, to regard $z_{0}^{*}$ as a constant, we aligned the traverse system with the generating line of the cone using a laser distance meter; the laser distance meter was mounted on the traversing system in the same way as the hot-wire probe and it ensured the distance from the cone surface remained in the range of \pm 10 micrometers (less than 0.1 in the nondimensional $z$ ) at different $x$ locations.

By choosing a specific voltage (say $E_{i}^{*}$ ) and using Fig. 19 and interpolating to get the corresponding rotational speed for the different heights $\left(\Omega_{i j}^{*}=2 \pi N_{i j} / 60\right)$, we know that for all heights we should have the same velocity. We illustrate this in Fig. 19 by $N_{i 1}^{*}, N_{i 2}^{*}, N_{i 3}^{*}$, and $N_{i 4}^{*}$. For each voltage $E_{i}^{*}$ we calculate the variance of the fitting to Eq. (A1) by varying $z_{0}^{*}$ in $1 \mu \mathrm{m}$ steps. We do that for all $j$ values and then calculate the total rms error by taking the square root of the sum of the variances. For the data in Fig. 19 we use voltages in the range $E_{i}^{*}=4.1$ to 5.0 Volt, with 0.1 Volt increments, i.e., ten different voltages. Each voltage can be obtained from different heights $z_{i j}^{*}$ and 


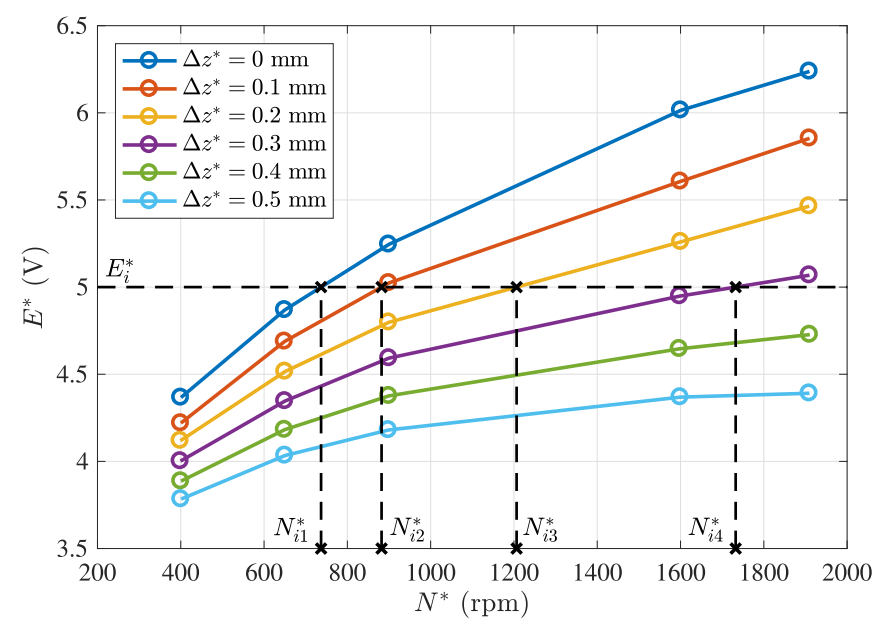

FIG. 19. Calibration voltages as a function of rotational speed for six different wall-normal positions. The figure also illustrates how, for a given $E_{i}^{*}$, we can determine the corresponding rotational speeds, $N_{i 1}^{*}$ to $N_{i 4}^{*}$.

corresponding different rotational rates $\Omega_{i j}^{*}$ that are interpolated from the data as shown in Fig. 19. Given $E_{i}^{*}$ is a function of velocity independent of position and angular velocity, it is now possible to obtain an estimate of the real distance from the wall. For a given $E_{i}^{*}$ we can estimate the velocity at each of the points $j\left(1 \leqslant j \leqslant n_{i}\right)$ for a given displacement $z_{0}^{*}$ from Eq. (A1) such that

$$
V_{i j}^{*}=\Omega_{i j}^{*} x^{*} \sin \psi f\left[\left(z_{i j}^{*}+z_{0}^{*}\right) / \delta_{v}^{*}\right] .
$$

However, since we do not know the value of $z_{0}^{*}$ we sweep $z_{0}^{*}$ over a certain range with small steps $(1 \mu \mathrm{m})$ and calculate $V_{i j}^{*}$ for each $z_{0}^{*}$. By calculating the average velocity for each value of $z_{0}^{*}$, we obtain an estimate of the velocity, $\overline{V_{i}^{*}\left(z_{0}^{*}\right)}$ at that value of $E_{i}^{*}$, and a corresponding measure of the deviation for each value of $z_{0}^{*}$ as

$$
\operatorname{Dev}_{i}^{*}\left(z_{0}^{*}\right)=\frac{1}{n_{i}} \sum_{j=1}^{n_{i}}\left[V_{i j}^{*}-\overline{V_{i}^{*}\left(z_{0}^{*}\right)}\right]^{2} .
$$

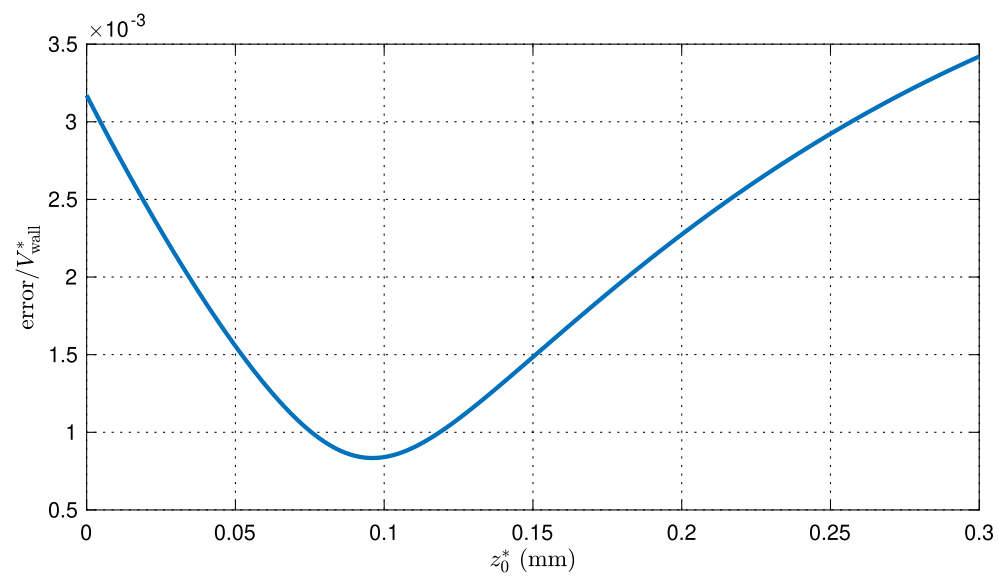

FIG. 20. Results of the optimization procedure to find the position relative to the wall. 

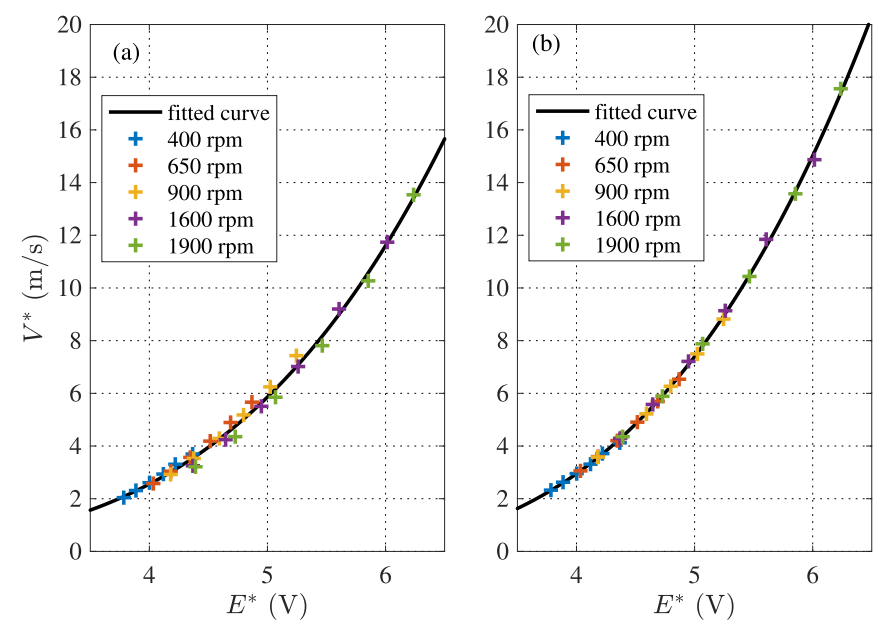

FIG. 21. Calibration curves including all data points in Fig. 19 where the optically determined wall distance is used for the points in (a), whereas the calibration curve using the optimized wall distance is shown in (b).

We do this operation for all values of $E_{i}^{*}$ that we have chosen $(1 \leqslant i \leqslant M)$ and calculate

$$
\operatorname{Dev}^{*}\left(z_{0}^{*}\right)=\frac{1}{M} \sum_{i=1}^{M} \operatorname{Dev}_{i}^{*}\left(z_{0}^{*}\right)
$$

Finally, we find the value of $z_{0}^{*}$ that gives the smallest total deviation (see Fig. 20, where we have used $M=10$ ) and assume that this displacement gives us the best estimate of the distance to the wall as $z_{m}^{*}+z_{0}^{*}$.
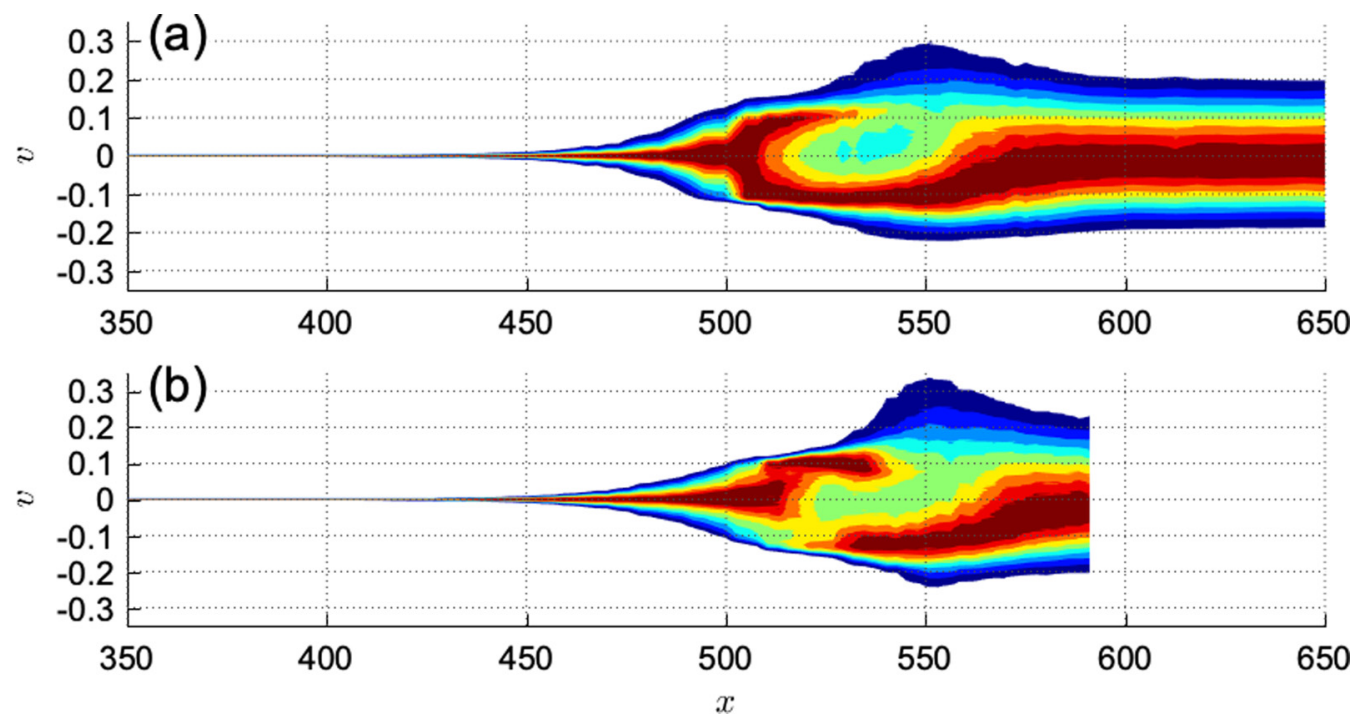

FIG. 22. The PDF of the azimuthal velocity fluctuation $v$ : (a) $60^{\circ}$ clean cone at $z=1.2,1800 \mathrm{rpm}$, (b) $60^{\circ}$ clean cone at $z=1.2,900 \mathrm{rpm}$. Filled contours indicate $10 \%, 20 \%, 30 \%, 40 \%, 50 \%, 60 \%, 70 \%$, $80 \%$, and $90 \%$ of the local maxima of PDF. 


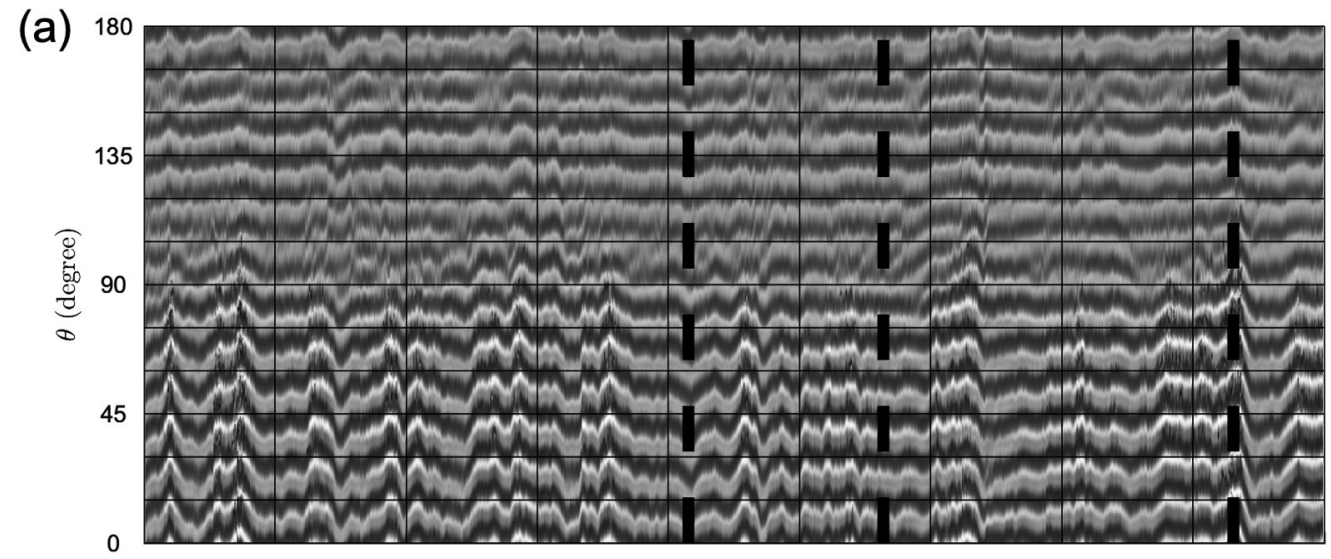

(b)

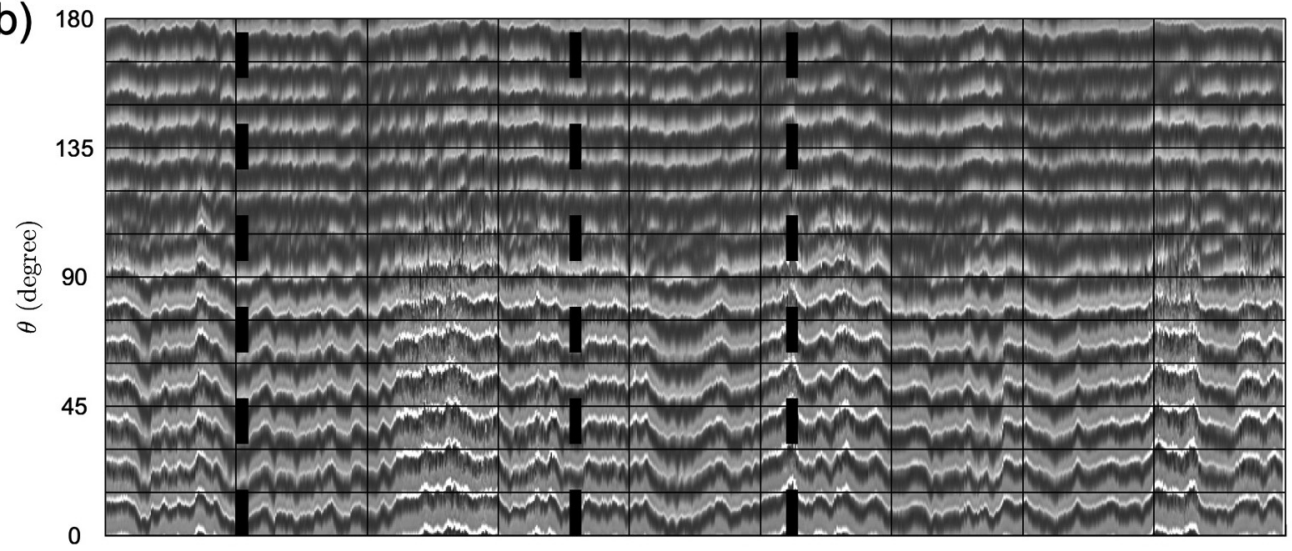

(c)

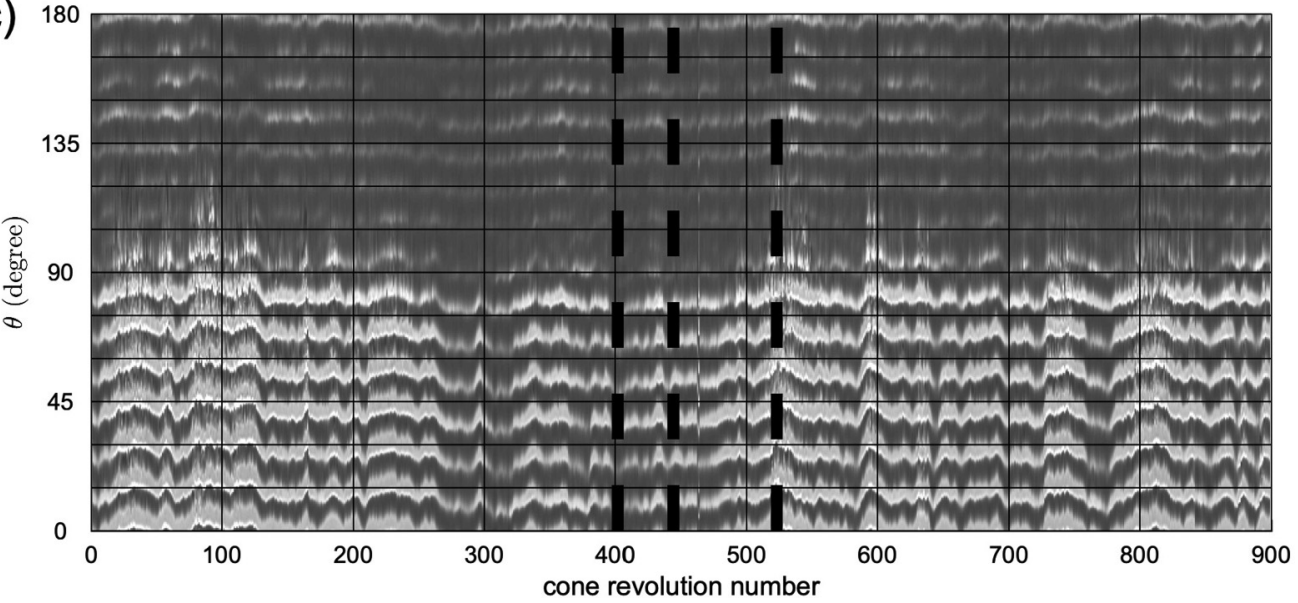

FIG. 23. Velocity fluctuation $v$ for the full sample population at $x=535$ on the clean cone (C in Figs. 5 and 7). The contours (dark and light indicate negative and positive, respectively) are scaled by three times the local rms of the azimuthal velocity $v_{\text {rms }}$, corresponding to (a) $\pm 3 v_{\text {rms }}= \pm 3.3 \times 10^{-1}$ at $z=1.2$; (b) $\pm 3 v_{\text {rms }}=$ $\pm 2.4 \times 10^{-1}$ at $z=2.4$; and (c) $\pm 3 v_{\text {rms }}= \pm 2.2 \times 10^{-1}$ at $z=3.2$. The three dashed lines from the left to right indicate where the vortex is shifted upstream, intermediate and downstream: the 415, 564, and 831 revolutions for (a), the 105, 359, and 524 revolutions for (b), and the 402, 444, and 523 revolutions for (c) are examined in Fig. 16. 
The result of the optimization procedure is found in Fig. 20, which shows a distinct error minimum. The preliminary optical determination of the wall position is seen to be off by approximately $96 \mu \mathrm{m}$. As can be seen, the rms error in velocity is less than $0.1 \%$ of the wall velocity.

The resulting calibration curves are shown in Fig. 21, both with the approximate, optically determined, wall position (left) and with the wall position determined from the optimization procedure (right). The line is obtained from a least-square fit of the modified King's law [25] given by

$$
V^{*}=k_{1}^{*}\left(E^{* 2}-E_{0}^{* 2}\right)^{1 / n}+k_{2}^{*}\left(E^{*}-E_{0}^{*}\right)^{1 / 2},
$$

where $E^{*}$ is the anemometer voltage at the velocity $V^{*}, E_{0}^{*}$ is the voltage at zero velocity taken when the cone is at rest, and $k_{1}^{*}, k_{2}^{*}$, and $n$ are calibration constants. According to the original King's law $n=0.50$ for an infinitely long cylinder, here we find a value of 0.48 .

This kind of method to calibrate hot wires against laminar boundary profiles can be used for different flows where the exact wall position is unknown. The methodology does not only give a calibration curve but also gives a good estimate of the wall position.

\section{APPENDIX B: EFFECT OF THE ROTATIONAL SPEED}

In this paper we present results from the 60 degree rotating cone with a rotation speed of $900 \mathrm{rpm}$. The instability and further development of the vortex structure should be solely dependent on the Reynolds number and not on the rotation rate per se. However, the rotation speed affects the viscous length scale of the flow $\left[\sqrt{\nu^{*} /\left(\Omega^{*} \sin \psi\right)}\right]$ so both the length $x^{*}$ along the generatrix as well as the height of any roughness, which is inevitable on the cone surface, in terms of the viscous length scale vary with the rotational speed. In Fig. 22, the PDF plots of the disturbance as function of $x$ for both the $1800 \mathrm{rpm}$ (a) and $900 \mathrm{rpm}$ (b) clean cone cases are shown; the latter shows the same data as in Fig. 5(b). It is clear from the figures that there is almost no difference between the two cases showing that the Reynolds number similarity holds as expected. It also shows that for these experiments the different nondimensional lengths from the apex to the rim of the cone do not influence the transition process.

\section{APPENDIX C: VORTEX SPLITTING AND MERGING ON THE CLEAN CONE}

In this section, we report the splitting and merging of the spiral vortices showing the velocity fluctuation $v$ in a similar way to that in Figs. 8 and 9. Figure 23 shows $v$ for the whole sample set at $x=535$ on the clean cone (to show the splitting and merging, only half of the cone is shown). In the area occupied by the vortices with lower amplitude $\left(\theta \gtrsim 75^{\circ}\right)$, some vortices merge into one vortex and one vortex splits into two vortices. In Fig. 23(a), for example, a vortex at $\theta=90^{\circ}$ splits into two around the 142nd revolution and two vortices merge into one at $\theta=105^{\circ}$ around the 624 th revolution. Because of the merging and splitting, the number of vortices changes through the revolutions. In contrast, vortices with larger amplitude $\left(\theta \lesssim 75^{\circ}\right)$ do not merge nor split although the vortex meandering occurs in a similar manner to the case with the artificial roughness elements (Figs. 8 and 9).

[1] N. Gregory, J. T. Stuart, and W. S. Walker, On the stability of three-dimensional boundary layers with application to the flow due to a rotating disk, Phil. Trans. R. Soc. A 248, 155 (1955).

[2] E. Crespo Del Arco, E. Serre, P. Bontoux, and B. E. Launder, Stability, transition and turbulence in rotating cavities, edited by M. Rahman, in Instability of Flows, Advances in Fluid Mechanics Vol. 41 (WIT Press, Ashurst, UK, 2005), pp. 141-195.

[3] R. Kappesser, R. Greif, and I. Cornet, Mass transfer to rotating cones, Appl. Sci. Res 28, 442 (1973). 
[4] F. Kreith, D. Ellis, and J. Giesing, An experimental investigation of the flow engendered by a rotating cone, Appl. Sci. Res., Sect. A 11, 430 (1963).

[5] Z. Hussain, S. J. Garrett, and S. O. Stephen, The centrifugal instability of the boundary-layer flow over slender rotating cones, J. Fluid Mech. 755, 274 (2014).

[6] T. von Kármán, Über laminare und turbulent Reibung, Z. Angew. Math. Mech. 1, 233 (1921).

[7] S. J. Garrett, Z. Hussain, and S. O. Stephen, The cross-flow instability of the boundary layer on a rotating cone, J. Fluid Mech. 622, 209 (2009).

[8] R. Kobayashi, Review: Laminar-to-turbulent transition of three-dimensional boundary layers on rotating bodies, ASME. J. Fluids Eng. 116, 200 (1994).

[9] S. J. Garrett and N. Peake, The absolute instability of the boundary layer on a rotating cone, Eur. J. Mech. B Fluids 26, 344 (2007).

[10] E. B. White and W. S. Saric, Secondary instability of crossflow vortices, J. Fluid Mech. 525, 275 (2005).

[11] K. J. Groot, J. Serpieri, F. Pinna, and M. Kotsonis, Secondary crossflow instability through global analysis of measured base flows, J. Fluid Mech. 846, 605 (2018).

[12] R. J. Lingwood, Absolute instability of the boundary layer on a rotating disk, J. Fluid Mech. 299, 17 (1995).

[13] R. J. Lingwood, An experimental study of absolute instability of the rotating-disk boundary-layer flow, J. Fluid Mech. 314, 373 (1996).

[14] F. Zoueshtiagh, R. Ali, A. J. Colley, P. J. Thomas, and P. W. Carpenter, Laminar-turbulent boundary-layer transition over a rough rotating disk, Phys. Fluids 15, 2441 (2003).

[15] S. Imayama, P. H. Alfredsson, and R. J. Lingwood, Experimental study of rotating-disk boundary-layer flow with surface roughness, J. Fluid Mech. 786, 5 (2016).

[16] E. Appelquist, P. Schlatter, P. H. Alfredsson, and R. J. Lingwood, Transition to turbulence in the rotatingdisk boundary-layer flow with stationary vortices, J. Fluid Mech. 836, 43 (2018).

[17] R. J. Lingwood and P. H. Alfredsson, Instabilities of the von Kármán Boundary Layer, Appl. Mech. Rev. 67, 030803 (2015).

[18] S. Imayama, P. H. Alfredsson, and R. J. Lingwood, On the laminar-turbulent transition of the rotating-disk flow: The role of absolute instability, J. Fluid Mech. 745, 132 (2014).

[19] R. Kobayashi, Y. Kohama, and Ch. Takamadate, Spiral vortices in boundary layer transition regime on a rotating disk, Acta Mech. 35, 71 (1980).

[20] S. Imayama, P. H. Alfredsson, and R. J. Lingwood, A new way to describe the transition characteristics of a rotating-disk boundary-layer flow, Phys. Fluids 24, 031701 (2012).

[21] S. Imayama, Studies of the rotating-disk boundary-layer flow, Ph.D. thesis, KTH Royal Institute of Technology, 2014.

[22] R. Kobayashi and H. Izumi, Boundary-layer transition on a rotating cone in still fluid, J. Fluid Mech. 127, 353 (1983).

[23] M. E. Siddiqui, V. Mukund, J. Scott, and B. Pier, Experimental characterization of transition region in rotating-disk boundary layer, Phys. Fluids 25, 034102 (2013).

[24] E. Appelquist, S. Imayama, P. H. Alfredsson, P. Schlatter, and R. J. Lingwood, Linear disturbances in the rotating-disk flow: A comparison between results from simulations, experiments and theory, Eur. J. Mech. B Fluids 55, 170 (2016).

[25] A. V. Johansson and P. H. Alfredsson, On the structure of turbulent channel flow, J. Fluid Mech. 122, 295 (1982). 